\title{
Renewal processes and fluctuation analysis of molecular motor stepping
}

\author{
Jaime E. Santos $\dagger$, Thomas Franosch $\dagger \ddagger$, Andrea Parmeggiani*, \\ and Erwin Frey $\ddagger, \S$ \\ $\dagger$ Hahn-Meitner Institut, Abteilung Theorie, Glienicker Str.100, D-14109 Berlin, \\ Germany \\ $\ddagger$ Arnold Sommerfeld Center and CeNS, Department of Physics, \\ Ludwig-Maximilians-Universität München, Theresienstrasse 37, D-80333 München, \\ Germany \\ * Laboratoire de Dynamique Moléculaire des Interactions Membranaires, UMR 5539 \\ CNRS/Université de Montpellier 2, Place Eugène Bataillon, 34095 Montpellier Cedex \\ 5, France.
}

\begin{abstract}
We present a systematic method of analysis of experiments performed with single motors proteins. The use of such a method should allow a more detailed description of the motor's chemical cycle through the precise fitting of the experimental data. We model the dynamics of a processive or rotary molecular motor using a renewal processes, in line with the work initiated by Svoboda, Mitra and Block. We apply a functional technique to compute different types of multiple-time correlation functions of the renewal process, which have applications to bead-assay experiments performed both with processive molecular motors, such as myosin $\mathrm{V}$ and kinesin, and rotary motors, such as F1-ATPase.
\end{abstract}

PACS numbers: 02.50.Ey,05.60.-k,05.40.-a

$\S$ To whom correspondence should be addressed (frey@lmu.de) 


\section{Introduction}

There has been a growing interest in the biology and biophysics communities in the study of the operation of motor proteins [1. Such biological agents are typically single enzymes that can act as thermodynamic engines, directly converting chemical energy into mechanical energy [2] through a chemical cycle which occurs at constant temperature [3]. Different classes of motor proteins are involved in biological processes such as cellular transport, cellular mitosis or muscle contraction, to cite a few examples of a plethora for which the presence of such agents is crucial.

In this article, we concentrate on two particular classes of motor proteins, namely linear processive molecular motors and rotary molecular motors. Linear processive motor proteins, of which myosin $\mathrm{V}$ and kinesin 4, 5. are important examples, catalyse the ATP hydrolysis reaction, ATP $\rightarrow \mathrm{ADP}+\mathrm{P}$, and use the energy so obtained to move along linear molecular tracks, carrying organelles or membrane patches with them and performing directed transport within cells with high efficiency [6]. These organelles are too massive for molecular diffusion to move them efficiently in a crowded environment such as the cytoplasm within the time scales relevant for biological processes. The molecular tracks, which are composed of actin in the case of myosin $\mathrm{V}$ and of tubulin in the case of kinesin, have a polar character, i.e. such motors can move in only one direction. A different processive molecular motor, dynein, moves along the tubulin track in the direction opposite to kinesin. Yet another example of a linear processive molecular motor is the enzyme RNA-polymerase; see [7] and references therein for a more complete discussion of the characteristics of this motor. Here it suffices to say that this enzyme moves along a DNA-strand, also using ATP hydrolysis as its energy source. However, the function of this enzyme is different from the motors described above, in that it does not perform molecular transport; instead it promotes the transcription of messengerRNA from the underlying DNA-strand on which it moves. Another significant difference is that the motion of this motor occurs in an heterogeneous medium such as the DNAstrand, rather than in homogeneous media such as the actin or tubulin molecular tracks.

The chemical cycle that results in the hydrolysis of one ATP molecule by the molecular motor is composed of several substeps, corresponding to changes in the internal conformation of the motor, accompanied by chemical reactions, e.g. ATP binding to the motor, ATP hydrolysis, ADP and $\mathrm{P}$ release, etc. Typically, one ATP hydrolysis is necessary for the motor to advance one step along the track, docking to the next available site in it, the size of such steps being around $35 \mathrm{~nm}$ in the case of myosin $\mathrm{V}$ and $8 \mathrm{~nm}$ in the case of kinesin [8, 9, 10, 11]. It should also be noted that the use of the word 'processive' to classify a molecular motor is limited to motors whose dwell time on the molecular track is much larger than the time for a complete chemical cycle, i.e. the typical time for a single step along the track.

Another class of molecular motors to which one may also apply some of the results

presented in this paper are the so-called rotary molecular motors, of which F1-ATPase is perhaps the most important example [12. This motor can also catalyse the ATP 
hydrolysis reaction and use the energy so obtained to generate the rotary motion of a shaft-like mechanism. The mechano-chemical cycle of such a motor is composed of three rotations of 120 degrees, determined by the symmetry of the molecule in question, each rotation being coupled to the hydrolysis of one ATP molecule, as described by the socalled "binding change mechanism" 13. If such a motor is coupled to the proton-driven Fo motor, the assembly of these two motors can either work as a proton pump or use a proton gradient to synthetise ATP, hence its name, ATPsynthase.

A great wealth of knowledge about the internal chemical cycle of linear processive molecular motors has been acquired through bead-assay experiments [14, 15, 9, 16, 4, 5. 17, 18. In such an experiment, performed in a fluid medium, a molecular motor is coupled to a dielectric bead of micrometric size, the size of such a sphere being nevertheless much larger than that of the motor protein. The bead can be manipulated through the use of optical or magnetic tweezers. In certain situations, one can, using an optical trap, perform experiments in which the molecular motor, moving along its track, is subjected to a known constant force of several pN. The study of the motion for different values of the applied force allows one to determine the force-velocity relation characteristic of a given motor. At a given value of the force, known as the stalling force, the motor usually decouples from the track (such decoupling can even occur in the absence of force, but it is a much rarer event in that case [19]) or it may move backwards along the track. Note that such experiments may be performed at distinct values of the applied load and also at different ATP concentrations.

More recently, Cappello et al. [20, 21] have considered an experimental apparatus containing a bead-motor assay that moves through the interference fringes of an evanescent light-wave that exists in the proximity of a microscope glass plate. The force which the electric field of such wave exerts on the bead is negligible, i.e. the experiment is performed in a zero-load condition. However, the bead still scatters photons of the evanescent field and the observation of such events may be used to track the bead's position with high spatial and time resolutions. In particular, such a method allows for measurements with time resolutions of $\mu s(\mathrm{MHz})$. In normal beadassays, the feedback mechanism used to keep the bead under constant force typically limits the time resolution to a few milliseconds $(\mathrm{kHz})$. Note that one may perform these measurements at different ATP concentrations, but one is always limited to work at zero external load, which constitutes both a strength (because it permits higher time resolutions) and a weakness of the method (because it limits the parameter range in which the system can be studied).

In essence, a clear qualitative picture has emerged from the different types of beadassay experiments, namely that a few of the substeps which compose the chemical cycle of a molecular motor (known in the literature as the rate-limiting steps) are of particularly long duration compared to the remaining substeps, and that the statistics of the motion of the molecular motor are, for the time resolutions available, essentially determined by the duration of such rate-limiting steps. Thus, the experimental study of the motion of linear processive molecular motors can provide valuable information 
concerning the nature and the duration of the rate-limiting steps under different load conditions or under different concentrations of ATP [18. There is experimental evidence that two rate-limiting steps are sufficient to describe the chemical cycle of myosin $\mathrm{V}$, namely ATP binding (by lowering enough the concentration of ATP it is always possible to make ATP binding to the motor a rate-limiting step) and ADP release [4, whereas three or four rate-limiting steps may be needed to describe the chemical cycle of kinesin, depending on the ATP concentration. It is not yet well understood to which chemical substeps these rates correspond [18].

The rotary movement of the $\gamma$ sub-unit of the F1-ATPase motor can be visualised by coupling such a motor to a microprobe, which can be either a fluorescent actin filament, a gold or polysterene bead, or a single fluorescent dye [12, 22]. The rotation of the probe can be recorded with a microscope and a CCD camera. It follows from such studies that the 360-degree rotation can be decomposed into three 120-degree substeps, each of which is in turn composed of two rate-limiting steps with approximately the same duration. These steps involve rotations of 90 and 30 degrees, respectively [23]. One should note that of the three different types of microprobe experiments referred, the use of a single fluorescent dye seems to be the most promising method of visualisation [12, 22, as it does not involve a perturbation of the motion of the molecule. In the other cases, the rotation of F1-ATPase is hindered by the large frictional coupling between the microprobe (actin filament or gold or polysterene bead) and the surrounding fluid.

The theoretical tool that we will be applying to the analysis of such experiments is the concept of renewal process. Such processes are ubiquitous in physics. These models have been successfully applied to describe the motion of processive molecular motors such as myosin $\mathrm{V}$ and kinesin [14, 20, 21], the statistics of detection of quantum particles [24, 25, 26] and persistence phenomena in kinetic Ising models [27, 28. Also, the results that relate to persistent phenomena have applications to studies of the volatility of financial markets [29]. The above list is not exhaustive.

Loosely speaking, a renewal process is a counting process where unit increments occur at random times. We will consider only independently distributed renewal processes, i.e. processes in which the probability distribution for the occurrence of the next increment (also known as the waiting-time distribution of the renewal process) depends only on the time elapsed since the occurrence of the last increment and not on the previous history of the counting process.

If one wishes to be more specific, one can say that the purpose of this article is to enumerate and classify a series of bead-assay experiments performed with linear processive molecular motors or with rotary molecular motors. These experiments are characterised by the fact that one can apply the simple (and exact) results obtained from the calculation of multiple-time correlation functions of renewal processes with an arbitrary waiting-time distribution to the analysis of the data obtained. In order to apply such simple models to linear processive motors, one represents a forward step of a molecular motor by an increment of the renewal process, whose waiting-time distribution is dependent on the number of rate-limiting steps of the motor. Such an approximation 
is justified if the molecular motor performs backward steps only infrequently within the time-window of observation, i.e. provided one is not working too close to the stall force or at ATP concentrations which are too low, when the rates for a forward or a backward step to occur become comparable and one needs to use a large time-window of observation. The application of these results to the dynamics of rotary motors involves the mapping of a rotation of the motor by a given angle to an increment of the renewal process, but such a mapping is more subtle (see below for details). Such a mapping can be justified provided that the motor performs backward rotations only infrequently within the time-window of observation, which is the case if one is working at high ATP concentrations.

The measurement of single-time correlation functions of the number of increments $N(T)$, which have occurred in a renewal process until a given time $T$, is a common practice in the context of the experimental study of linear processive molecular motors. The measurement of these quantities was first undertaken by Svoboda and coworkers in their experiments performed with the linear processive molecular motor kinesin [14]. These authors have considered the behaviour of the first and second moments of $N(T)$, i.e. $\langle N(T)\rangle$ and $\left\langle N^{2}(T)\right\rangle$, where the averaging is taken over different realisations of the experiment. Such correlation functions contain information concerning both the number of rate-limiting steps in a chemical cycle and the characteristic rates pertaining to such steps. However, such single-time correlation functions do not fully characterise the motor's chemical cycle, in particular in the case of motors whose cycles are composed of many rate-limiting steps, like kinesin. The measurement of multiple-time correlation functions can provide additional valuable information in such a case [20, 21].

We employ a method based on the use of the probability-generating functional [30, 31 to compute multiple-time correlation functions of a renewal process. For simplicity, and due to its experimental relevance, we explicitly compute the meansquare deviation of the number of increments that occur between time $t_{2}$ and a later time $t_{1}$. This correlation function is mathematically defined as $\left\langle\left(N\left(t_{1}\right)-N\left(t_{2}\right)\right)^{2}\right\rangle-$ $\left\langle N\left(t_{1}\right)-N\left(t_{2}\right)\right\rangle^{2}$, where the times $t_{2}$ and $t_{1}$ are large compared to the typical time of a single chemical cycle. We show that this function contains additional information concerning the rate-limiting steps of the chemical process, information which cannot be extracted from single-time correlation functions. We also briefly indicate how the computation of higher-order correlation functions can be performed. Furthermore, we explicitly compute the density-density correlation function for a single motor, which was considered in the experiments of Cappello and co-workers [20, 21]. Interestingly enough, one can show that in a certain limiting case, such a function is identical to the spin-spin correlation function considered by Godreche and Luck [27] in their study of persistence phenomena in kinetic Ising models. Furthermore, the class of spin models obtained in this particular limit (to which the model studied by Godreche and Luck belongs) may be relevant for the experimental study of rotary molecular motors, and hence it is also discussed here.

The structure of this paper is as follows: in the next section, we present the general 
results obtained and relate such results to the relevant experiments. In section 3. we provide a mathematical introduction to renewal processes, following [30] and introduce the probability-generating functional of the renewal process, together with some related quantities whose usefullness will become apparent in section 4 . In section 4, we provide a general derivation of the results presented in section 2, concerning multiple-time correlation functions of a renewal process, including the mean-square deviation and the density-density correlation functions mentioned above, whose expressions we will explicitly compute in the asymptotic regime of large times. A reader whose primary interest is not mathematics may skip sections 3 and 4 without loss of continuity with respect to the remainder of the paper. Finally, in section 5, we present our conclusions and provide a brief outlook of the experimental work that we believe can be carried in this field using the results that we have derived. In the appendices, we discuss two simple examples of waiting-time distributions for which the quantities discussed in the main text can be computed outside the asymptotic regime.

\section{General discussion}

The most elementary quantity one can measure in a bead-assay experiment is the average displacement of a motor $\langle x(T)\rangle$ up to time $T$ over many runs. The simplest model for such a motor is that $x(T)=N(T) d$, where $N(T)$ is an integer variable, updated at random intervals with a given distribution $f(\zeta)$ (a renewal process), and $d$ is the motor's step size. In the limit of large time, Blackwell's renewal theorem [32] guaranties that $\langle x(T)\rangle=T d /\langle\tau\rangle$ asymptotically, where $\langle\tau\rangle=\int_{0}^{\infty} d \zeta \zeta f(\zeta)$ is the average waiting time of the distribution and corresponds to the mean duration time (or turnover time) of a single chemical cycle.

The simplest choice one can take for $f(\zeta)$ is the exponential distribution $f(\zeta)=$ $e^{-\zeta / \tau} / \tau$. In this case, $\langle\tau\rangle=\tau$. In their discussion of bead-motor assay experiments performed on the processive motor kinesin, Svoboda, Mitra and Block 14 have examined the more complicated situation where the waiting-time distribution $f(\zeta)$ is given by the convolution of a finite number $\mathcal{M}$ of simple Poisson processes. Each of these processes, which occur in series with typical times $\tau_{1}, \cdots, \tau_{\mathcal{M}}$, is supposed to represent a rate-limiting step of the motor's chemical cycle. In such a case, the average waiting time is given by

$$
\langle\tau\rangle=\sum_{i=1}^{\mathcal{M}} \tau_{i} .
$$

Such a model is in good agreement with experiments where one measures the distance travelled by the molecular motor alone. If one wishes to resolve the chemical cycle substeps and its associated pathways, e.g. through the use of cryoelectron microscopy [33, 5], or by using bead-motor assays where lateral or forward loads are applied to the motor [18, one needs to make use of more involved models [34, 35, 5, 36, 37, 38, 39, 40, 41] in order to interpret such experiments. 
Svoboda et al. 14 have also introduced the concept of the randomness coefficient $r$, characteristic of a single molecular motor. Given that the quantity $\left\langle x^{2}(T)\right\rangle-\langle x(T)\rangle^{2}$ is the mean square deviation of the distance travelled by the motor, $r$ is defined as

$$
r=\lim _{T \rightarrow \infty} \frac{\left\langle x^{2}(T)\right\rangle-\langle x(T)\rangle^{2}}{\langle x(T)\rangle d} .
$$

The randomness coefficient, being the ratio of the mean-square deviation of distance travelled by the motor to the average distance travelled itself, represents a measure of the deviation of the motors stepping from a deterministic motion, which would occur if $r=0$. Also, note that $r$ is chosen to be dimensionless [42]. For the simple Poisson process, $\left\langle x^{2}(T)\right\rangle-\langle x(T)\rangle^{2}=T d^{2} / \tau$, i.e. $r=1$. For a renewal process composed of $\mathcal{M}$ rate-limiting steps [43], one can show [14] that the randomness parameter is given by

$$
r=\frac{\sum_{i=1}^{\mathcal{M}} \tau_{i}^{2}}{\left(\sum_{i=1}^{\mathcal{M}} \tau_{i}\right)^{2}} .
$$

It follows from (3) that if $\mathcal{M}>1, r<1$. For a renewal process composed of $\mathcal{M}$ ratelimiting steps, $r \geq 1 / \mathcal{M}$, the equality being obtained when all rates are equal. In the limit of an infinite number of substeps whose characteristic time tends to zero, $r=0$ and the motor performs a deterministic motion.

The measurement of the randomness parameter, which is robust against thermal noise [14] or the influence of the initial conditions (i.e. of the experimental set up), is a powerful experimental tool that can be used to rule out a proposed chemical cycle, if such a cycle contains too small a number of rate-limiting steps. For example, a measurement of $r<1 / 2$ indicates that at least three rate-limiting steps are needed to describe the motor's chemical cycle [4].

Since the chemical cycle of myosin $\mathrm{V}$ appears to be composed of only two ratelimiting steps, the joint measurement of $r$ and of the average distance travelled by the motor at large times is sufficient to determine the value of the typical times $\tau_{1}$ and $\tau_{2}$ associated to each substep. However, if one is considering the experimental study of motors with more than two rate-limiting steps in their cycle, such as kinesin, the measurement of these two quantities is not equivalent to the complete determination of the characteristic times $\tau_{1}, \ldots, \tau_{\mathcal{M}}(\mathcal{M}>2)$.

The statistical analysis of step duration using such bead-motors assays, which allows for the direct extraction of the waiting-time distribution $f(\zeta)$, was also considered by several authors [9, 4]. By fitting $f(\zeta)$ with an appropriate convolution of exponential functions, one can obtain the values of all the characteristic times $\tau_{1}, \ldots, \tau_{\mathcal{M}}$ for arbitrary $\mathcal{M}$. However, such a technique requires the substitution of a signal with a rich structure (the individual trajectories of the motor as a function of time) by a function in steps, which leads to an effective truncation of the data, because possible substeps within the chemical cycle are erased. Furthermore, such a technique requires a resolution of individual steps, which is not always feasible.

One is thus led to consider the information provided by multiple-time correlation functions. The simplest quantity one can consider is the average number of steps given 
by the motor between times $t_{2}$ and $t_{1}$, i.e. the correlation function $\left\langle N\left(t_{1}\right)-N\left(t_{2}\right)\right\rangle$, which for large times $t_{1}, t_{2} \rightarrow \infty$ behaves as $\left(t_{1}-t_{2}\right) /\langle\tau\rangle$, and which obviously does not carry any new information. Its measurement yields, as above, the value of the turnover time $\langle\tau\rangle$ of the chemical cycle.

The same cannot be said of the mean-square deviation of this quantity, i.e. the connected correlation function $\left\langle\left(N\left(t_{1}\right)-N\left(t_{2}\right)\right)^{2}\right\rangle_{\text {conn }}=\left\langle\left(N\left(t_{1}\right)-N\left(t_{2}\right)\right)^{2}\right\rangle-\left\langle N\left(t_{1}\right)-\right.$ $\left.N\left(t_{2}\right)\right\rangle^{2}$. If one were to take $t_{2}=0$, this correlation function would reduce to the mean square deviation of the distance travelled by the motor up to time $t_{1}$, introduced above, and from which one can extract the randomness parameter, as defined in (2). However, there are good reasons to consider instead the opposite limit in which both $t_{1}, t_{2} \rightarrow \infty$, with $t=t_{1}-t_{2}$ kept finite, as in this limit the correlation function becomes dependent only on $t$, i.e. one recovers a form of time-translation invariance (we will write the correlation function in this $\operatorname{limit}$ as $\left.\mathcal{C}(t)=\lim _{t_{1}, t_{2} \rightarrow \infty}\left\langle\left(N\left(t_{1}\right)-N\left(t_{2}\right)\right)^{2}\right\rangle_{\text {conn }}\right)$. Such a limit is of experimental relevance, since such a correlation function becomes independent of the initial conditions, which can change from one experimental realisation to the other. Furthermore, its measurement contains additional information concerning the waitingtime distribution, rather than just the value of the randomness parameter, as we will now discuss. Finally, such a measurement, just like the measurement of the average number of steps $\left\langle N\left(t_{1}\right)-N\left(t_{2}\right)\right\rangle$, requires only the ensemble averaging of the data and is not plagued by the limitations one encounters if one tries to measure $f(\zeta)$ directly, as described above.

In our treatment, we will consider a renewal process with an arbitrary waiting-time distribution, rather than the special choice made by Svoboda et al., since the study of the general case does not involve a more complicated analysis. However, we will always indicate the results for this particular waiting-time distribution, given its experimental importance.

It will be shown in section 4 that this correlation function displays the following behaviour in the limit of small and large time differences $t=t_{1}-t_{2}$,

$$
\mathcal{C}(t)=\left\{\begin{array}{c}
\frac{t}{\langle\tau\rangle} \text { if } t \ll\langle\tau\rangle \\
\frac{r}{\langle\tau\rangle} t+C \text { if } t \gg\langle\tau\rangle
\end{array},\right.
$$

with $r=\frac{\left\langle\tau^{2}\right\rangle-\langle\tau\rangle^{2}}{\langle\tau\rangle^{2}}$ and $C=\frac{\left\langle\tau^{2}\right\rangle^{2}}{2\langle\tau\rangle^{4}}-\frac{\left\langle\tau^{3}\right\rangle}{3\langle\tau\rangle^{3}}$, where $\left\langle\tau^{2}\right\rangle=\int_{0}^{\infty} d \zeta \zeta^{2} f(\zeta)$ and $\left\langle\tau^{3}\right\rangle=$ $\int_{0}^{\infty} d \zeta \zeta^{3} f(\zeta)$. Please note that the result just quoted for $r$, the randomness parameter, is a generalisation of equation (3) to the case of general waiting-time distribution $f(\zeta)$ [27. The constant $C$ can be written in terms of $r$ and of the connected third-moment of the waiting-time distribution, $\delta=\frac{\left\langle(\tau-\langle\tau\rangle)^{3}\right\rangle}{2\langle\tau\rangle^{3}}$, as

$$
C=\frac{1}{6}+\frac{1}{2} r^{2}-\frac{2}{3} \delta
$$

In the case in which $f(\zeta)$ is given by the convolution of $\mathcal{M}$ Poisson processes, as considered by Svoboda et al., one can show (see section 4) that $r$ reduces to equation 
(31). In this case, $\delta=\frac{\sum_{i=1}^{\mathcal{M}} \tau_{i}^{3}}{\left(\sum_{i=1}^{\mathcal{M}} \tau_{i}\right)^{3}}$. The constant $C$ is exactly zero for a simple Poisson process.

As we will show in Appendix A, one can compute $\mathcal{C}(t)$ for arbitrary values of $t$, rather than just the asymptotic limits given by (4), for processes whose waiting-time distribution is given by the convolution of two or three Poisson processes, i.e. if $\mathcal{M}=2,3$ (the case $\mathcal{M}=1$ is trivial, see section 44). Such computations are explicitly performed because of the importance of such processes for the experimental study of myosin $\mathrm{V}$ and kinesin. We plot the results of these calculations below, in figures 1 and 2. respectively, with the choices $\tau_{1}=\tau_{2}=1 / 2$ for the case of a renewal process composed of two ratelimiting steps and $\tau_{1}=\tau_{2}=\tau_{3}=1 / 3$ for the case of a renewal process composed of three rate-limiting steps. In both cases, $\langle\tau\rangle=1$ (in arbitrary units), with $r=1 / 2$ in the first case and $r=1 / 3$ in the second case. It is seen that the two functions have the correct asymptotic limits at short and large times, as given by equation (44).

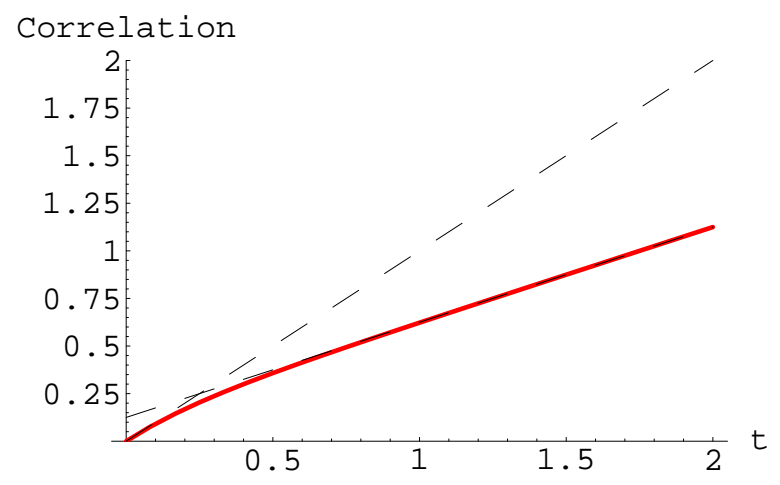

Figure 1. Mean-square deviation of the number of steps in the regime of large times (red curve), for a motor with two rate-limiting steps. Also shown are the linear regimes at small and large time $t$.

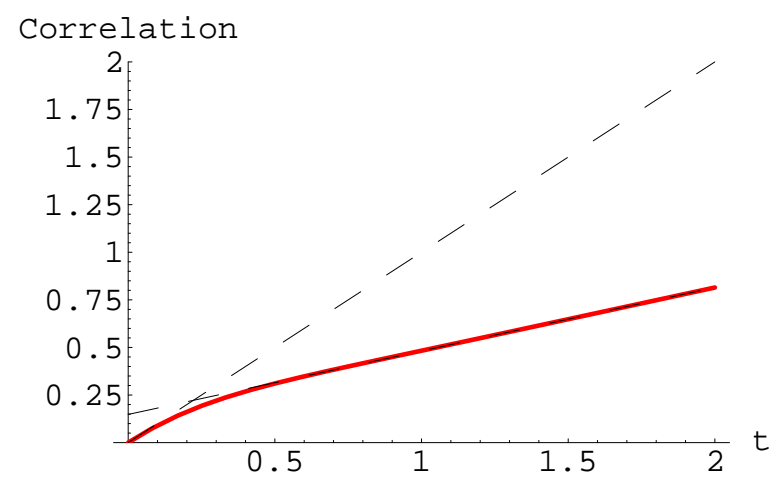

Figure 2. Mean-square deviation of the number of steps in the regime of large times (red curve), for a motor with three rate-limiting steps. Also shown are the linear regimes at small and large time $t$.

As stated above, the measurement of the average distance travelled by the motor permits one to determine the turnover time $\langle\tau\rangle$. Once this quantity is known, one 
can use the measurement of $\mathcal{C}(t)$ at large times to determine both the randomness coefficient $r$ (which is given by the slope of the straight line multipled by $\langle\tau\rangle$ ), as well as the constant $C$ which is related to the second and third moment of $f(\zeta)$ [45. For a motor whose chemical cycle is composed of two rate-limiting steps, the constant $C$ is given by $C=\frac{2 \tau_{1}^{2} \tau_{2}^{2}}{\left(\tau_{1}+\tau_{2}\right)^{4}}$. The measurement of $C$ does not provide any new information in this case, but it may provide a way to check or to improve the results obtained from the joint measurement of the average distance travelled by the motor and of the randomness parameter $r$. On the other hand, in the case of a motor whose chemical cycle is composed of three rate-limiting steps, the measurement of the total distance travelled by the motor, which permits one to determine the total turnover time $\langle\tau\rangle=\tau_{1}+\tau_{2}+\tau_{3}$, of $r$ and of $C=\frac{2}{\left(\tau_{1}+\tau_{2}+\tau_{3}\right)^{4}}\left(\tau_{1}^{2} \tau_{2}^{2}+\tau_{1}^{2} \tau_{2} \tau_{3}+\tau_{1} \tau_{2}^{2} \tau_{3}+\tau_{1}^{2} \tau_{3}^{2}+\tau_{1} \tau_{2} \tau_{3}^{2}+\tau_{2}^{2} \tau_{3}^{2}\right)$ can be used to determine the three time-constants $\tau_{1}, \tau_{2}$ and $\tau_{3}$, through a fit of the experimental results. Such a measurement may be particularly useful in experiments with kinesin and for concentrations of ATP for which ATP binding is not a rate-limiting step, because in such a case the chemical cycle of kinesin appears to be composed of three rate-limiting steps [18.

Another example of a multiple-time correlation function which can be measured experimentally is the density-density correlator of an ensemble of independent molecular motors, defined as $S\left(q, t_{1}, t_{2}\right)=\left\langle e^{-i q d\left(N\left(t_{1}\right)-N\left(t_{2}\right)\right)}\right\rangle$, where $q$ has the dimensions of a wave-vector and $d$ is the motor's step size. This quantity is the Fourier transform of the probability for the motor to move by a distance $n \times d$ between time $t_{2}$ and $t_{1}$. It was directly measured in the experiments of Cappello and coworkers [46], where the beadmotor assay moves through the interference fringes of an evanescent wave. In such an experiment, $q=2 \pi / \Lambda$, where $\Lambda$ is the period of the mask used to create the interference pattern [20, 21]. In the long time limit $t_{2}, t_{1} \rightarrow \infty, S\left(q, t_{1}, t_{2}\right)$ becomes solely dependent on the time difference $t=t_{1}-t_{2}$, in which case one simply writes $S(q, t)$. One can show (see section 4) that the Laplace transform of such a function, $\tilde{S}(q, s)=\int_{0}^{\infty} d t e^{-s t} S(q, t)$, is given, for a general distribution $f(\zeta)$ with a finite average time $\langle\tau\rangle$, by

$$
\tilde{S}(q, s)=\frac{1}{s}\left(1+\frac{\left(e^{-i q d}-1\right)(1-\tilde{f}(s))}{\langle\tau\rangle s\left(1-e^{-i q d} \tilde{f}(s)\right)}\right),
$$

where $\tilde{f}(s)$ is the Laplace transform of $f(\zeta)$. Note that in the experiments of Cappello and co-workers, this function was taken to be a simple exponential distribution. The equation above generalises their result to the case of a general waiting-time distribution.

One can relate the Fourier transform of $S(q, t), S(q, \omega)=\int_{-\infty}^{+\infty} d t e^{i \omega t} S(q, t)$ to its Laplace transform by $S(q, \omega)=2 \operatorname{Re} \tilde{S}(q, s=-i \omega)$. The Fourier transform is more amenable to computation from the measured data than the Laplace transform, or can otherwise be directly measured. One can show from such a relation and from equation (6) that $S(q, \omega)$ is given by

$$
S(q, \omega)=\frac{2(1-\cos (q d))\left(1-|\tilde{f}(-i \omega)|^{2}\right)}{\omega^{2}\langle\tau\rangle\left|1-e^{-i q d} \tilde{f}(-i \omega)\right|^{2}} .
$$


If $f(\zeta)$ is a simple exponential distribution, one obtains from (7),

$$
S(q, \omega)=\frac{2 \tau(1-\cos (q d))}{(\omega \tau-\sin (q d))^{2}+(1-\cos (q d))^{2}},
$$

which becomes the result for asymetric diffusion in the continuum limit $q d \ll 1$, i.e. one obtains in this limit

$$
S(q, \omega)=\frac{2 D q^{2}}{(\omega-q v)^{2}+D^{2} q^{4}},
$$

where we have introduced the motor's velocity $v=d / \tau$ and the motor's diffusion constant $D=d^{2} / 2 \tau$, valid for a Poisson process, since equation (9) was derived from (8). For a renewal process with waiting-time distribution $f(\zeta)$, one can still approximate $S(q, \omega)$ by (9) if the Laplace transform $\tilde{f}(s)$ is analytic at $s=0$, i.e. if all moments of the distribution $f(\zeta)$ exist. In such a case, $v=d /\langle\tau\rangle$ and $D=d^{2} r / 2\langle\tau\rangle$. Such approximation is valid at low-frequencies compared to the total turnover rate and at low-momenta compared to the inverse step size, i.e. if $\omega\langle\tau\rangle \ll 1$ and $q d \ll 1$. In other words, the motor behaves in such a limit as a Brownian particle, characterized by the two parameters $v$ and $D$ [21], as one would expect. In Appendix B, we will explicitly compute $S(q, \omega)$ outside this asymptotic region for the cases in which $f(\zeta)$ is given by the convolution of two or three Poisson processes, which corresponds to a chemical cycle composed of two or three rate-limiting steps. Again, such calculations are carried through because of the experimental significance of these two cases for the study of myosin $\mathrm{V}$ and of kinesin, since a measurement of $S(q, t)$ and the subsequent computation of $S(q, \omega)$ would permit the extraction of the relevant time constants by fitting the measured $S(q, \omega)$ with the corresponding expression, appropriate for the given number of rate-limiting steps, as given in Appendix B 47. Note that in order to perform such a fitting, one should first have a qualitative understanding of the nature of the chemical cycle, in particular of the number of rate-limiting steps.

Interestingly enough, one may also use the results obtained above for $\tilde{S}(q, s)$ to interpret experiments performed with rotary molecular motors, such as F1-ATPase [12, 13. In this case, one assimilates the rotary motion of the probe attached to the motor to the motion of classical spin in two dimensions (an XY model), with unit length and $Q$ internal states, where $Q$ is an integer, such that the spin rotates around the $z$-axis in one direction by an angle equal to $2 \pi / Q$. Such a model may be appropriately referred to as a 'random-clock'.

In more mathematical terms, one defines the 2-dimensional vector variable $\vec{\sigma}_{T}=$ $(\cos (2 \pi N(T) / Q), \sin (2 \pi N(T) / Q))$, where $N(T)$ is given by a renewal process with an arbitrary waiting-time distribution $f(\zeta)$. Such a vector rotates in the 2-dimensional circle by an angle equal to $2 \pi / Q$, with the waiting-time distribution of the renewal process $f(\zeta)$. After $Q$ rotations, the spin returns to its original state. It can be easily seen that the spin-spin correlation function of such a model is given by $\left\langle\vec{\sigma}_{t_{1}} \cdot \vec{\sigma}_{t_{2}}\right\rangle=\operatorname{Re} S\left(q=2 \pi / Q, t_{1}, t_{2}\right)$, where $S\left(q, t_{1}, t_{2}\right)$ is the density-density correlator defined above for the renewal process with waiting-time distribution $f(\zeta)$ and $d=1$, since $q$ is here an angular variable. In the long-time limit, $t_{1}, t_{2} \rightarrow \infty$, where this 
function is solely dependent on $t=t_{1}-t_{2}$, one can read the Laplace transform of such a quantity from equation (6). This transform is given by

$$
\mathcal{L}\left\langle\vec{\sigma}_{t_{1}} \cdot \vec{\sigma}_{t_{2}}\right\rangle=\operatorname{Re}\left[\frac{1}{s}\left(1+\frac{\left(e^{-2 \pi i / Q}-1\right)(1-\tilde{f}(s))}{\langle\tau\rangle s\left(1-e^{-2 \pi i / Q} \tilde{f}(s)\right)}\right)\right] .
$$

If we were to take $Q=2$, the model would become that of an Ising spin variable and equation (10) reduces to the result obtained by Godreche and Luck [27]. In effect, the computation of $\tilde{S}(q, s)$, as given by (6) , can also be obtained from their results. We will use a different method to derive such an expression.

In the case of F1-ATPase, the symmetry of the motor molecule implies that $Q=3$, i.e. the motor rotates by an angle of 120 degrees and requires three such rotations to complete its cycle. If one were to assume that the waiting-time distribution for each of these 120 degrees rotations was given by a simple Poisson process, it becomes trivial to invert the Laplace transform given in equation (10) and one obtains (with $t_{1}>t_{2}$ )

$$
\left\langle\vec{\sigma}_{t_{1}} \cdot \vec{\sigma}_{t_{2}}\right\rangle=\cos \left(\frac{\sqrt{3}\left(t_{1}-t_{2}\right)}{2 \tau}\right) \exp \left(-\frac{3\left(t_{1}-t_{2}\right)}{2 \tau}\right),
$$

i.e., the spin-spin correlation function, despite the irreversible character of the underlying spin model, displays an oscillatory, albeit overdamped, behaviour. In fact, such behaviour is always present if $Q>2$. Its origin is trivial, being traceable to the circular character of the motion. Nevertheless, the study of the motion may provide valuable information concerning the chemical cycle. In equation (11), such information is encoded in the turnover time $\tau$.

In reality, the 120-degree rotation performed by F1-ATPase is composed of two rate-limiting substeps, the first substep corresponding to a 90-degree rotation and the second substep to a 30-degree rotation [12. This implies that $f(\zeta)$ is given by the convolution of two Poisson processes. In such a case, it is still possible to invert the Laplace transform given by equation (11), but the resulting expression for the spinspin correlation function in the time domain is rather cumbersome and it is preferable to work with its Fourier transform instead. However, there is one particular instance in which the spin-spin correlation function in the time domain acquires a particularly simple form, namely when the time-constants $\tau_{1}$ and $\tau_{2}$ are equal, i.e. $\tau_{1}=\tau_{2}=\tau / 2$. One obtains for the spin-spin correlation function in this case, the result (with $t_{1}>t_{2}$ )

$$
\left\langle\vec{\sigma}_{t_{1}} \cdot \vec{\sigma}_{t_{2}}\right\rangle=\cos \left(\frac{\sqrt{3}\left(t_{1}-t_{2}\right)}{\tau}\right)\left(\frac{3}{4} e^{-\frac{\left(t_{1}-t_{2}\right)}{\tau}}+\frac{1}{4} e^{-\frac{3\left(t_{1}-t_{2}\right)}{\tau}}\right),
$$

whose form is clearly different from the one given in equation (111), as the period of the oscillations is now shorter than its decay-time, i.e. the oscillations are no longer overdamped. This property may actually permit the experimental observation of such oscillations. We leave the derivation of equation (12) to Appendix C. We plot below, in figure 3, the two functions given by (11) and (12) for comparison, in terms of the variable $t=t_{1}-t_{2}$.

The derivation of the expression of the Fourier transform of the spin-spin correlation function, for a general choice of the two time constants $\tau_{1}$ and $\tau_{2}$, is also left to 


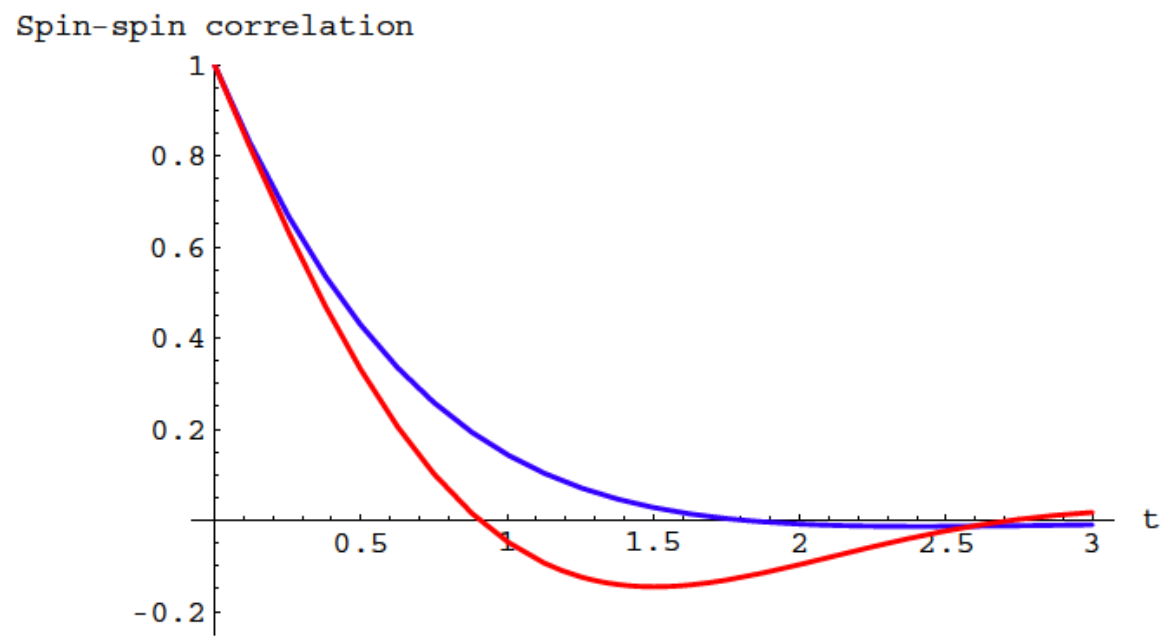

Figure 3. Spin-spin correlation functions as given by equations (11) (blue curve) and (12) (red curve). It is seen that the second function decays more slowly than the first and it also oscillates more rapidly.

Appendix C. Below, in figure 4, we plot such a function for the three distinct choices $\tau_{1}=1, \tau_{2}=0$, which is just the Fourier transform of equation (11]), $\tau_{1}=4 / 5, \tau_{2}=1 / 5$, and $\tau_{1}=\tau_{2}=1 / 2$, which is the Fourier transform of equation (12). The choice of units is such that the total turnover time $\tau=\tau_{1}+\tau_{2}=1$. Note that the difference between the two extreme cases $\tau_{1}=1, \tau_{2}=0$ and $\tau_{1}=\tau_{2}=1 / 2$ is more pronounced if the functions are plotted in Fourier space (figure 4) rather than in time-domain (figure (3). The experimental situation is closer to the third case considered, i.e. the two timeconstants are approximately equal to $1 \mathrm{~ms}$ [12]. Here again, the measurement of the spin-spin correlation, followed by a Fourier transformation of the signal, is the preferred method for the extraction of the chemical cycle's time constants, since one can fit the experimentally measured signal with the result of equation (‥3), which then yields $\tau_{1}$ and $\tau_{2}$.

As we have mentioned above, the measurement of the spin-spin correlation function should ideally be performed using a fluorescent dye, since such a probe does not affect the rotation of F1-ATPase. The visualisation of the single fluorophore, coupled to the rotating $\gamma$ sub-unit of F1-ATPase, is performed by excitation of the fluorophore with circular polarized light and by measuring the intensities, $H$ and $V$, of the light emitted by the molecule along two perpendicular directions of polarisation in a dual-view apparatus [22]. The geometry of the experimental setup is such that the polarisation of the observed light is parallel to the dipolar moment of the fluorophore, and is therefore determined by the instantaneous angle of rotation $\theta(T)=\frac{2 \pi}{3} N(T)$, of the $\gamma$ sub-unit with respect to the fixed $\alpha \beta$ sub-units [22], where we take $N(T)$ to be given by a renewal process, as above. Hence, one concludes that $H(T) \propto \cos ^{2}\left(\frac{2 \pi}{3} N(T)+\phi\right), V(T) \propto \sin ^{2}\left(\frac{2 \pi}{3} N(T)+\phi\right)$, where $\phi$ is the angle between the horizontal polarisation direction and the nearest polarisation 


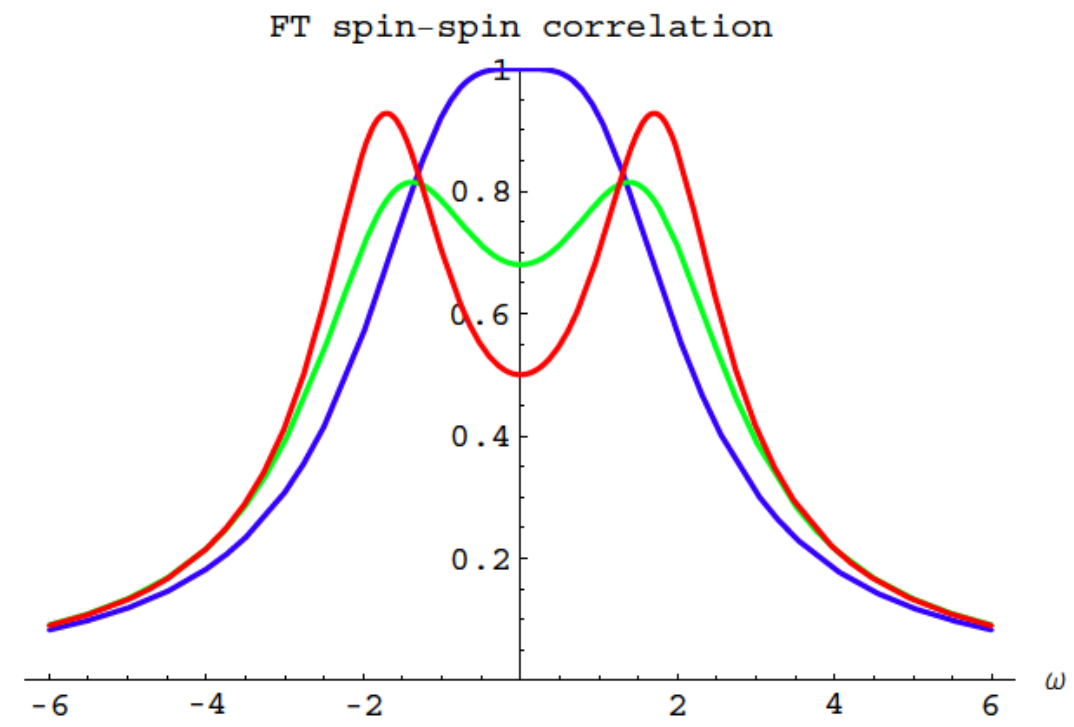

Figure 4. Fourier transform of the spin-spin correlation function for a renewal process composed of two Poisson processes. The three cases shown correspond to the choices, $\tau_{1}=1, \tau_{2}=0$ (blue curve), $\tau_{1}=4 / 5, \tau_{2}=1 / 5$ (green curve) and $\tau_{1}=\tau_{2}=1 / 2$ (red curve). Note that the area under the curve is the same for all plots.

direction along which light is emitted, with $\phi=18^{\circ}$ in the experiments of Adachi and co-workers 22].

With such definitions in hand, we define $h\left(t_{2}\right)= \pm \sqrt{\frac{H\left(t_{2}\right)}{H\left(t_{2}\right)+V\left(t_{2}\right)}}, v\left(t_{2}\right)=$ $\pm \sqrt{\frac{V\left(t_{2}\right)}{H\left(t_{2}\right)+V\left(t_{2}\right)}}$, the sign in these definitions being determined by the value of the instantaneous polarisation $P\left(t_{2}\right)=h^{2}\left(t_{2}\right)-v^{2}\left(t_{2}\right)$ [48. Using the same definition for $h\left(t_{1}\right), v\left(t_{1}\right)$ at a later time $t_{1}$, one concludes that

$$
\left\langle\vec{\sigma}_{t_{1}} \cdot \vec{\sigma}_{t_{2}}\right\rangle=\left\langle\cos \left[2 \pi\left(N\left(t_{1}\right)-N\left(t_{2}\right)\right) / 3\right]\right\rangle=\left\langle h\left(t_{1}\right) h\left(t_{2}\right)\right\rangle+\left\langle v\left(t_{1}\right) v\left(t_{2}\right)\right\rangle,
$$

an equality that shows that the spin-spin correlation function can be related to the average value of a directly measurable quantity. One should note that the experiments of Adachi et al. 22] were performed at low ATP concentrations where ATP binding is a rate-limiting step, with typical binding times of the order of seconds. If one wishes to measure the characteristic times $\tau_{1}$ and $\tau_{2}$, one needs to perform the experiment at much higher ATP concentrations and with a time-resolution which is three orders of magnitude higher than in the experiment of Adachi and co-workers $\left(\tau_{1} \approx \tau_{2} \approx 1 \mathrm{~ms}\right)$. Achieving such a resolution may constitute an insurmountable practical problem, even if the method theoretically permits an unlimited time-resolution [49].

\section{Definition of the renewal process and associated quantities}

A renewal process is defined by a random variable $N(u)$ that is incremented by one unit at random times $u=T_{1}<T_{2}<\ldots<T_{\mathcal{N}}$ where, in the simplest case and to which we will attain, the length of the intervals $T_{1}, T_{2}-T_{1}, \ldots, T_{\mathcal{N}}-T_{\mathcal{N}-1}$ is taken from a 
given waiting time distribution $f(\zeta)$ such that $f(\zeta)$ is positive and $\int_{0}^{\infty} d \zeta f(\zeta)=1$ (one assumes that the process starts at $u=0$ with $N(0)=0$ ). Mathematically, this can be written as

$$
N(u)=n \text { if } T_{n} \leq u<T_{n+1},
$$

with $T_{0}=0$. If one defines $F_{k}(T)$ to be the probability that at least $k$ increments have occurred up to time $T$ irrespective of whether more increments have occurred or not, then it is easy to show 32 that $F_{k}(T)$ is given by

$$
\begin{aligned}
& F_{0}(T)=1 \\
& F_{k}(T)=\int_{0}^{T} d \zeta F_{k-1}(T-\zeta) f(\zeta) k>0 .
\end{aligned}
$$

One may equally regard the renewal process as a totally asymetric random-walk of a structureless particle, in which case one speaks of the probability of observing at least $k$ forward steps of such a particle up to time $T$. As discussed above, this particle is supposed to represent a molecular motor, with the waiting time-distribution $f(\zeta)$ being determined by the internal chemical cycle of the motor.

In particular, it follows from this definition that $F_{1}(T)=\int_{0}^{T} d \zeta f(\zeta)$ is the cumulative probability to observe an increment of $N(u)$ in the time interval $[0, T$. The probability that exactly $k$ increments have occurred up to time $T$ is then given by

$$
P_{k}(T)=F_{k}(T)-F_{k+1}(T) .
$$

The average number of increments of $N(u)$ (or the average number of steps of the particle) up to time $T$, which we designate by $m(T)$, is given by $m(T)=\langle N(T)\rangle=$ $\sum_{k=0}^{\infty} k P_{k}(T)$. Using equation (17) for $P_{k}(T)$ and expressing $F_{k+1}(T)$ in terms of $F_{k}(u)$ at earlier times through equation (16), it follows that $m(T)$ necessarily obeys the integral equation

$$
m(T)=F_{1}(T)+\int_{0}^{T} d \zeta m(T-\zeta) f(\zeta)
$$

which is known in the literature as the 'renewal equation', $m(T)$ being called the 'renewal function'. For reasons that will become clear below, we prefer to work with the derivative of $m(T)$ and we write $l(T)=m^{\prime}(T)$, which we will also call the renewal function. Differentiating the above equation with respect to $T$ and given that $m(0)=0$, one obtains

$$
l(T)=f(T)+\int_{0}^{T} d \zeta l(T-\zeta) f(\zeta)
$$

i.e. the equation has the same form as (18), but the non-homogeneous term is now given by $f(T)$ rather than by $F_{1}(T)$. From the above equation, it also follows that $l(0)=f(0)$. If one considers a Poisson process, where $f(\zeta)=e^{-\zeta / \tau} / \tau$, it is trivial to verify that $l(T)=1 / \tau$ is the solution of equation (19) and one has $m(T)=T / \tau$, i.e. the average number of steps of the walker increases linearly with time. For more 
general forms of the waiting-time distribution, the renewal equation can still be solved by Laplace transformation, and one obtains

$$
\tilde{l}(s)=\frac{\tilde{f}(s)}{1-\tilde{f}(s)},
$$

where $\tilde{l}(s)$ and $\tilde{f}(s)$ are, respectively, the Laplace transforms of $l(T)$ and of $f(T)$. In order to obtain $l(T)$ from this expression, one needs to invert the Laplace transform, an operation one can only perform in a limited number of cases. Nevertheless, Blackwell's renewal theorem [32] asserts that provided that $\langle\tau\rangle=\int_{0}^{\infty} d \zeta \zeta f(\zeta)$ is finite, then $l(T)=\frac{1}{\langle\tau\rangle}$ asymptotically at large time $T$.

Having defined the random process that will be the object of our study, we now proceed to define the quantities we wish to compute. We will first consider the restriction of the renewal process given above such that exactly $\mathcal{N}$ increments have occurred between time $u=0$ and time $u=T$, i.e. the times of the increments are such that $0<T_{1}<T_{2}<\ldots<T_{\mathcal{N}}<T<T_{\mathcal{N}+1}$ and we will consider correlation functions of the random variable 31$]$

$$
\mathcal{J}(u)=\sum_{i=1}^{\mathcal{N}} g\left(u-T_{i}\right)
$$

where $0 \leq u<T$ and where $g(u)$ is a function with at most a finite number of discontinuities. If $\mathcal{N}=0$, we define $\mathcal{J}(u) \equiv 0$ for all $0 \leq u<T$. Note that $N(u)=\sum_{i=1}^{\mathcal{N}} \theta\left(u-T_{i}\right)$, where $\theta(u)=0$ if $u<0, \theta(u)=1$ if $u \geq 0$, is of this form, and therefore the number of increments $N(u)$ (or the number of steps which the particle has given) corresponds to a particular case of (21) in which one takes $g(u)=\theta(u)$. Thus, we wish to determine the value of the correlation functions $\left\langle\mathcal{J}\left(t_{1}\right) \mathcal{J}\left(t_{2}\right) \ldots \mathcal{J}\left(t_{m}\right)\right\rangle_{\mathcal{N}}$, where $m \geq 1$ and where the averaging is over the distribution of the times $T_{1}$ to $T_{\mathcal{N}}$. For simplicity, we will restrict ourselves to $m=1$ and $m=2$ in the particular examples discussed, though the formalism is valid for arbitrary $m$.

If one defines the probability-generating functional $Z_{\mathcal{N}}[j]$ as

$$
Z_{\mathcal{N}}[j]=\left\langle\exp \left(i \int_{0}^{T} d x j(x) \mathcal{J}(x)\right)\right\rangle_{\mathcal{N}},
$$

where the averaging is performed over the increment times $T_{1}, \ldots, T_{\mathcal{N}}$, then it follows that the computation of $Z_{\mathcal{N}}[j]$ is equivalent to the computation of all correlation functions, which can be obtained by functionally differentiating $Z_{\mathcal{N}}[j]$ with respect to $j$. Note that we choose the normalisation of $Z_{\mathcal{N}}[j]$ to be given by

$$
Z_{\mathcal{N}}[j=0]=P_{\mathcal{N}}(T),
$$

where $P_{\mathcal{N}}(T)$ is the probability of occurrence of exactly $\mathcal{N}$ increments up to time $T$, introduced above.

One can now generalise the concept of probability-generating functional to situations in which the total number of increments is not fixed by introducing the (grand-canonical) functional 31]

$$
\mathcal{Z}[j]=\sum_{\mathcal{N}=0}^{\infty} Z_{\mathcal{N}}[j] .
$$


The functional $\mathcal{Z}[j]$ can again be functionally differentiated with respect to $j$ in order to obtain the relevant correlation function. Note that if one takes $j=0$, one obtains

$$
\mathcal{Z}[j=0]=\sum_{\mathcal{N}=0}^{\infty} P_{\mathcal{N}}(T)=1
$$

i.e. the probability-generating functional is equal to one due to normalisation of the total probability. This result justifies the normalisation chosen above for $Z_{\mathcal{N}}[j]$. In the next section, we will develop a technique which will allow us to compute the correlators of $\mathcal{J}(u)$ order by order.

\section{Integral equation for the probability-generating functional and associated multiple-time correlation functions}

We first consider the case in which exactly $\mathcal{N}>0$ increments have occurred up to time $T$. The exclusive probability density (also known as Janoussi local density) [50, 30] for the increments to occur at times $T_{1}<T_{2}<\ldots<T_{\mathcal{N}}<T$ is given by

$$
p_{\mathcal{N}}\left(T_{1}, T_{2}, \ldots, T_{\mathcal{N}}\right)=P_{0}\left(T-T_{\mathcal{N}}\right) f\left(T_{\mathcal{N}}-T_{\mathcal{N}-1}\right) \ldots f\left(T_{2}-T_{1}\right) f\left(T_{1}\right)
$$

where $P_{0}\left(T-T_{\mathcal{N}}\right)=1-\int_{0}^{T-T_{\mathcal{N}}} d \zeta f(\zeta)$ is the probability that no increment has ocurred between $T_{\mathcal{N}}$ and $T$, as given by equation (17) (with $k=0$ ). The probability-generating functional $Z_{\mathcal{N}}[j]$ is given in terms of this quantity by

$$
\begin{aligned}
Z_{\mathcal{N}}[j]= & \int_{0}^{T} d T_{\mathcal{N}} \int_{0}^{T_{\mathcal{N}}} d T_{\mathcal{N}-1} \ldots \int_{0}^{T_{2}} d T_{1} p_{\mathcal{N}}\left(T_{1}, T_{2}, \ldots, T_{\mathcal{N}}\right) e^{i \int_{0}^{T} d x j(x) \mathcal{J}(x)} \\
= & \int_{0}^{T} d T_{\mathcal{N}} P_{0}\left(T-T_{\mathcal{N}}\right) e^{i \int_{0}^{T} d x j(x) g\left(x-T_{\mathcal{N}}\right)} \\
& \int_{0}^{T_{\mathcal{N}}} d T_{\mathcal{N}-1} f\left(T_{\mathcal{N}}-T_{\mathcal{N}-1}\right) e^{i \int_{0}^{T} d x j(x) g\left(x-T_{\mathcal{N}-1}\right)} \ldots \\
& \int_{0}^{T_{2}} d T_{1} f\left(T_{2}-T_{1}\right) e^{i \int_{0}^{T} d x j(x) g\left(x-T_{1}\right)} f\left(T_{1}\right)
\end{aligned}
$$

where we have used the identity $e^{i \int_{0}^{T} d x j(x) \mathcal{J}(x)}=\prod_{l=1}^{N} e^{i \int_{0}^{T} d x j(x) g\left(x-T_{l}\right)}$. Note that if $j(x)=0$, the functional $Z_{\mathcal{N}}[j=0]$ reduces to $P_{\mathcal{N}}(T)$, as stated above. This equation can be written in a more condensed form if one introduces the following set of functionals, defined recursively by

$$
\begin{aligned}
& l_{0}(j, u)=f(u) \\
& l_{\mathcal{N}}(j, u)=\int_{0}^{u} d v f(u-v) e^{i \int_{0}^{T} d x j(x) g(x-v)} l_{\mathcal{N}-1}(j, v) .
\end{aligned}
$$

Using this notation, it is then easy to see that one can write the functionals $Z_{\mathcal{N}}[j]$ in the form

$$
\begin{aligned}
& Z_{0}[j]=P_{0}(T) \\
& Z_{\mathcal{N}}[j]=\int_{0}^{T} d u P_{0}(T-u) e^{i \int_{0}^{T} d x j(x) g(x-u)} l_{\mathcal{N}-1}(j, u) \text { if } \mathcal{N}>0 .
\end{aligned}
$$


Since we wish to compute the functional $\mathcal{Z}[j]$, we need to performed the summation defined in equation (24). If one now introduces the functional

$$
l(j, u)=\sum_{\mathcal{N}=0}^{\infty} l_{\mathcal{N}}(j, u),
$$

then one can show from equation (28) that $l(j, u)$ obeys the integral equation

$$
l(j, u)=f(u)+\int_{0}^{u} d v f(u-v) e^{i \int_{0}^{T} d x j(x) g(x-v)} l(j, v) .
$$

Now, using the recursion relation (29), it is easy to show that $\mathcal{Z}[j]$ can be expressed in terms of $l(j, u)$ by

$$
\mathcal{Z}[j]=P_{0}(T)+\int_{0}^{T} d u P_{0}(T-u) e^{i \int_{0}^{T} d x j(x) g(x-u)} l(j, u) .
$$

As they stand, equations (31) and (32) are of little use, since it is usually not possible to solve (31) for $l(j, u)$. However, if one takes $j(x)=0$ in equation (31), one obtains, after changing the integration variable from $v \rightarrow u-v$, the equation

$$
l(0, u)=f(u)+\int_{0}^{u} d v l(0, u-v) f(v),
$$

with the initial condition $l(0,0)=f(0)$. But this is precisely equation (19) for the renewal function $l(u)$, with the same initial condition. Therefore, we conclude that $l(0, u)=l(u)$, a result which justifies the notation we are using. Now, we express $f(u)$ and $f(u-v)$ in equation (31) in terms of $l(u)$, using (33). After interchanging the limits of integration in the resulting double integral and using (31), one obtains the integral equation

$$
l(j, u)=l(u)+\int_{0}^{u} d v l(u-v)\left(e^{i \int_{0}^{T} d x j(x) g(x-v)}-1\right) l(j, v),
$$

which shows explicitly that $l(j, u)$ reduces to $l(u)$ when $j(x)=0$. Now, substituting $P_{0}(T)=1-\int_{0}^{T} d u f(u), P_{0}(T-u)=1-\int_{u}^{T} d v f(v-u)$ in equation (32) and interchanging the limits of integration in the resulting double integral, one obtains, after using equation (31)

$$
\mathcal{Z}[j]=1+\int_{0}^{T} d u\left(e^{i \int_{0}^{T} d x j(x) g(x-u)}-1\right) l(j, u),
$$

which shows explicitly that $\mathcal{Z}[j=0]=1$. Equations (34) and (35) are the main results of this section. In fact, these two equations are equivalent to the expansion of the probability-generating functional $\mathcal{Z}[j]$ in terms of the conditional probability densities for a renewal process, see [30] for details. The above form is more convenient for explicit calculations, as shown below.

Before we discuss the general solution of (34), let us apply our results to compute the functional $\mathcal{Z}[j]$ for the particular case of the simple Poisson process. Given that $l(u)=1 / \tau$, one concludes, substituting this result in equation (34), that $l(j, u)$ obeys the integral equation

$$
l(j, u)=\frac{1}{\tau}+\int_{0}^{u} \frac{d v}{\tau}\left(e^{i \int_{0}^{T} d x j(x) g(x-v)}-1\right) l(j, v) .
$$


Differentiating this equation with respect to $u$, one obtains the first-order linear differential equation

$$
\frac{d l(j, u)}{d u}=\frac{1}{\tau}\left(e^{i \int_{0}^{T} d x j(x) g(x-u)}-1\right) l(j, u) .
$$

with the initial condition $l(j, 0)=1 / \tau$. Such an equation can be quickly integrated by separation of variables, yielding the solution

$$
l(j, u)=\frac{1}{\tau} \exp \left(\int_{0}^{u} \frac{d v}{\tau}\left(e^{i \int_{0}^{T} d x j(x) g(x-v)}-1\right)\right) .
$$

This solution, when substituted in equation (35), yields

$$
\mathcal{Z}[j]=\exp \left(\int_{0}^{T} \frac{d u}{\tau}\left(e^{i \int_{0}^{T} d x j(x) g(x-u)}-1\right)\right),
$$

which is a well known result, being given in, e.g. [31]. However, this is in fact the only case for which a closed expression for $\mathcal{Z}[j]$ can be found, as one can no longer reduce the integral equation (34) to a first-order differential equation if one chooses a different waiting-time distribution. Neither can one solve (34) using a Laplace transform because the source term $e^{i \int_{0}^{T} d x j(x) g(x-v)}$ in (34) is not time-translation invariant. Such difficulties stem from the fact that equation (34) is a Volterra integral equation for which no analytical solution is known in the general case.

Nevertheless, one can show that all correlation functions can be obtained from equations (34) and (35) in a closed form, provided one knows the renewal function $l(u)$. This point can be made clearer through the discussion of two examples which we will use extensively in our applications of renewal processes to the study of the dynamics of molecular motors. We wish to compute the average value $\left\langle\mathcal{J}\left(t_{1}\right)\right\rangle$ and the two-point correlation function $\left\langle\mathcal{J}\left(t_{1}\right) \mathcal{J}\left(t_{2}\right)\right\rangle$. In the first case, one has

$$
\begin{aligned}
\left\langle\mathcal{J}\left(t_{1}\right)\right\rangle= & -\left.i \frac{\delta \mathcal{Z}[j]}{\delta j\left(t_{1}\right)}\right|_{j=0} \\
= & \left(\int_{0}^{T} d u g\left(t_{1}-u\right) e^{i \int_{0}^{T} d x j(x) g(x-u)} l(j, u)\right. \\
& \left.-i \int_{0}^{T} d u\left(e^{i \int_{0}^{T} d x j(x) g(x-u)}-1\right) \frac{\delta l(j, u)}{\delta j\left(t_{1}\right)}\right)_{j=0} \\
= & \int_{0}^{T} d u g\left(t_{1}-u\right) l(u),
\end{aligned}
$$

since the second term is zero if $j(x)=0$ and $l(j=0, u)=l(u)$. Therefore, the average value $\left\langle\mathcal{J}\left(t_{1}\right)\right\rangle$ can be expressed as an integral involving the known functions $g(u)$ and $l(u)$. In particular, if $\mathcal{J}\left(t_{1}\right)=N\left(t_{1}\right)$, which is the number of increments which has occurred up to time $t_{1}$ or the displacement of the particle up to time $t_{1}$, then one has $g(u)=\theta(u)$ and one obtains

$$
\left\langle N\left(t_{1}\right)\right\rangle=\int_{0}^{t_{1}} d u l(u)=m\left(t_{1}\right)
$$

which is just the definition of the renewal function $m\left(t_{1}\right)$ given in the previous section. 
The procedure used to compute the two point correlator $\left\langle\mathcal{J}\left(t_{1}\right) \mathcal{J}\left(t_{2}\right)\right\rangle$ is analogous, but it also involves the functional derivative $\frac{\delta l(j, u)}{\delta j\left(t_{1,2}\right)}$. One has

$$
\begin{aligned}
\left\langle\mathcal{J}\left(t_{1}\right) \mathcal{J}\left(t_{2}\right)\right\rangle= & -\left.\frac{\delta^{2} \mathcal{Z}[j]}{\delta j\left(t_{1}\right) \delta j\left(t_{2}\right)}\right|_{j=0}=\int_{0}^{T} d u g\left(t_{1}-u\right) g\left(t_{2}-u\right) l(u) \\
& -\left.i \int_{0}^{T} d u g\left(t_{1}-u\right) \frac{\delta l(j, u)}{\delta j\left(t_{2}\right)}\right|_{j=0}-\left.i \int_{0}^{T} d u g\left(t_{2}-u\right) \frac{\delta l(j, u)}{\delta j\left(t_{1}\right)}\right|_{j=0},(42)
\end{aligned}
$$

since the term which involves the functional derivative $\frac{\delta^{2} l(j, u)}{\delta j\left(t_{1}\right) \delta j\left(t_{2}\right)}$ is zero if $j(x)=0$. Now, in order to compute the functional derivative $\frac{\delta l(j, u)}{\delta j\left(t_{1}\right)}$ at $j(x)=0$, one functionally differentiates equation (34) with respect to $j\left(t_{1}\right)$ and sets $j(x)=0$. One obtains

$$
\begin{aligned}
\left.\frac{\delta l(j, u)}{\delta j\left(t_{1}\right)}\right|_{j=0}= & i\left(\int_{0}^{u} d v l(u-v) g\left(t_{1}-v\right) e^{i \int_{0}^{T} d x j(x) g(x-v)} l(j, v)\right. \\
& \left.+\int_{0}^{u} d v l(u-v)\left(e^{i \int_{0}^{T} d x j(x) g(x-v)}-1\right) \frac{\delta l(j, v)}{\delta j\left(t_{1}\right)}\right)_{j=0} \\
= & i \int_{0}^{t} d v l(u-v) g\left(t_{1}-v\right) l(v)
\end{aligned}
$$

since the second term is again zero if $j(x)=0$ and $l(j=0, v)=l(v)$. The expression for the derivative $\frac{\delta l(j, u)}{\delta j\left(t_{2}\right)}$ is simply obtained from equation (43) by interchanging $t_{1}$ by $t_{2}$.

It should be clear from equation (40) that in order to compute the $m$-th variational derivative of $\mathcal{Z}[j]$ at $j(x)=0$, one needs only the derivative of order $m-1$ of $l(j, u)$, because the term which involves the $m$-th derivative of $l(j, u)$ is zero in that case, since $e^{i \int_{0}^{T} d x j(x) g(x-u)}-1=0$ if $j(x)=0$. More importantly, it should also follow from the structure of equation (43) that in order to compute the $m$-th variational derivative of $l(j, u)$ at $j(x)=0$, one only needs the derivative of order $m-1$ of $l(j, u)$, because the term which involves the $m$-th derivative of $l(j, u)$ is zero for the same reason. This implies that one can express such variational derivatives in terms of quantities which were previously calculated, assuming that we know the function $l(u)$. Thus, the solution of equation (19) is sufficient to compute all correlation functions in a recursive manner, see [30] for a rigorous proof.

Finally, one can obtain a closed expression for $\left\langle\mathcal{J}\left(t_{1}\right) \mathcal{J}\left(t_{2}\right)\right\rangle$ by substituting the result for $\left.\frac{\delta l(j, u)}{\delta j\left(t_{1,2}\right)}\right|_{j=0}$ given in equation (43) in equation (42). One has that $\left\langle\mathcal{J}\left(t_{1}\right) \mathcal{J}\left(t_{2}\right)\right\rangle$ is given by

$$
\begin{aligned}
\left\langle\mathcal{J}\left(t_{1}\right) \mathcal{J}\left(t_{2}\right)\right\rangle= & \int_{0}^{T} d u g\left(t_{1}-u\right) g\left(t_{2}-u\right) l(u) \\
& +\int_{0}^{T} d u g\left(t_{1}-u\right) \int_{0}^{u} d v l(u-v) g\left(t_{2}-v\right) l(v) \\
& +\int_{0}^{T} d u g\left(t_{2}-u\right) \int_{0}^{u} d v l(u-v) g\left(t_{1}-v\right) l(v) .
\end{aligned}
$$

If $\mathcal{J}\left(t_{1}\right)=N\left(t_{1}\right), \mathcal{J}\left(t_{2}\right)=N\left(t_{2}\right)$, then $g(u)=\theta(u)$ and we obtain for the correlation function $\left\langle N\left(t_{1}\right) N\left(t_{2}\right)\right\rangle$, the result

$$
\left\langle N\left(t_{1}\right) N\left(t_{2}\right)\right\rangle=\int_{0}^{\min \left(t_{1}, t_{2}\right)} d u l(u)+\int_{0}^{t_{1}} d u \int_{0}^{\min \left(u, t_{2}\right)} d v l(u-v) l(v)
$$




$$
+\int_{0}^{t_{2}} d u \int_{0}^{\min \left(u, t_{1}\right)} d v l(u-v) l(v) .
$$

In the context of the study of molecular motors, it is more appropriate to consider the mean-square deviation of the displacement of the particle between time $t_{2}$ and $t_{1}$ $\left(t_{1}>t_{2}\right)$, i.e. the correlation function $\left\langle\left(N\left(t_{1}\right)-N\left(t_{2}\right)\right)^{2}\right\rangle_{\text {conn }}=\left\langle\left(N\left(t_{1}\right)-N\left(t_{2}\right)\right)^{2}\right\rangle-$ $\left\langle N\left(t_{1}\right)-N\left(t_{2}\right)\right\rangle^{2}$, as explained in section 2. If we use equations (41) and (45), we obtain, after some trivial manipulations,

$$
\left\langle\left(N\left(t_{1}\right)-N\left(t_{2}\right)\right)^{2}\right\rangle_{\mathrm{conn}}=\int_{t_{2}}^{t_{1}} d u l(u)+2 \int_{t_{2}}^{t_{1}} d u l(u) \int_{u}^{t_{1}} d v(l(v-u)-l(v)) .
$$

In the case of a Poisson process, $l(u)=1 / \tau$ and this expression reduces to the first term, i.e. we obtain $\left\langle\left(N\left(t_{1}\right)-N\left(t_{2}\right)\right)^{2}\right\rangle_{\text {conn }}=\left(t_{1}-t_{2}\right) / \tau$, which is a well-known result for the simple Poisson process. The expression for the full functional obtained above in equation (39) allows one to compute correlation functions of any order, but only in this simple case.

A particular important limit of (46) is the one in which $t_{2}, t_{1} \rightarrow \infty$, but in which the time difference $t=t_{1}-t_{2}$ is kept finite, since $\mathcal{C}(t)=\lim _{t_{1}, t_{2} \rightarrow \infty}\left\langle\left(N\left(t_{1}\right)-N\left(t_{2}\right)\right)^{2}\right\rangle_{\text {conn }}$ becomes a function of $t$ only, i.e. one recovers a form of time translation invariance. In this limit, $l(u) \rightarrow \frac{1}{\langle\tau\rangle}$ and one obtains, substituting this result above, the following result for $\mathcal{C}(t)$

$$
\mathcal{C}(t)=\frac{t}{\langle\tau\rangle}+\frac{2}{\langle\tau\rangle} \int_{0}^{t} d u(t-u)\left(l(u)-\frac{1}{\langle\tau\rangle}\right) .
$$

If $t \ll\langle\tau\rangle$, it is easy to see that the second term of this equation is at least $O\left(t^{2}\right)$ and therefore $\mathcal{C}(t) \approx \frac{t}{\langle\tau\rangle}$, if $t \ll\langle\tau\rangle$. This observation justifies the first line of equation (4). In order to extract the long-time behaviour of $\mathcal{C}(t)$, one needs to consider instead the Laplace transform $\tilde{\mathcal{C}}(s)$ of $\mathcal{C}(t)$. One has, from equation (47),

$$
\tilde{\mathcal{C}}(s)=\frac{1}{\langle\tau\rangle s^{2}}\left(\frac{1+\tilde{f}(s)}{1-\tilde{f}(s)}-\frac{2}{\langle\tau\rangle s}\right),
$$

where we have used equation (20) to express $\tilde{l}(s)$ in terms of $\tilde{f}(s)$. If $\tilde{f}(s)$ is analytic a $s=0$, i.e. if all moments of the distribution $f(\zeta)$ exist, one can write, for small $s$ $\tilde{f}(s)=1-\langle\tau\rangle s+\frac{\left\langle\tau^{2}\right\rangle}{2 !} s^{2}-\frac{\left\langle\tau^{3}\right\rangle}{3 !} s^{3}+\ldots$ Performing a Taylor expansion of (48) at $s=0$ using such a result for $\tilde{f}(s)$ and keeping only the most divergent terms, one obtains

$$
\tilde{\mathcal{C}}(s) \approx \frac{r}{\langle\tau\rangle s^{2}}+\frac{C}{s},
$$

with $r=\frac{\left\langle\tau^{2}\right\rangle-\langle\tau\rangle^{2}}{\langle\tau\rangle^{2}}$ and $C=\frac{\left\langle\tau^{2}\right\rangle^{2}}{2\langle\tau\rangle^{4}}-\frac{\left\langle\tau^{3}\right\rangle}{3\langle\tau\rangle^{3}}$, where $\left\langle\tau^{2}\right\rangle=\int_{0}^{\infty} d \zeta \zeta^{2} f(\zeta)$ and $\left\langle\tau^{3}\right\rangle=$ $\int_{0}^{\infty} d \zeta \zeta^{3} f(\zeta)$. Furthermore, one can write $C$ in the form given by equation (5), using the definitions of the connected second- and third-moment $r$ and $\delta$, given above. The behaviour of $\mathcal{C}(t)$ for $t \gg\langle\tau\rangle$ is determined by the behaviour of the Laplace transform $\tilde{\mathcal{C}}(s)$ at small $s$, as given by (49) and one can directly identify the coefficients of the 
most divergent terms of $\mathcal{C}(t)$ from those in equation (49). One thus concludes that $\mathcal{C}(t)$ is given, in this limit, by the second line of equation (44).

Let us now consider the case in which the waiting-time distribution of the renewal process is given by a convolution of $\mathcal{M}$ Poisson processes, each of which is supposed to represent a rate-limiting step of the molecular motor. Mathematically, this is expressed by saying that such function, which we denote by $f_{\mathcal{M}}(\zeta)$, is given recursively by

$$
f_{\mathcal{M}}(\zeta)=\int_{0}^{\zeta} \frac{d \eta}{\tau_{\mathcal{M}}} e^{-\frac{\zeta-\eta}{\tau_{\mathcal{M}}}} f_{\mathcal{M}-1}(\eta) \text { if } \mathcal{M}>1
$$

where $f_{\mathcal{M}-1}(\eta)$ is the waiting-time distribution of a process with $\mathcal{M}-1$ rate-limiting steps and $f_{1}(\zeta)=e^{-\zeta / \tau_{1}} / \tau_{1}$. Given that the Laplace transform of a single Poisson process is given by $\tilde{f}_{1}(s)=\left(1+\tau_{1} s\right)^{-1}$, one concludes from equation (150) and the convolution theorem that the Laplace transform of $f_{\mathcal{M}}(\zeta)$ is given by

$$
\tilde{f}_{\mathcal{M}}(s)=\prod_{k=1}^{\mathcal{M}} \frac{1}{1+s \tau_{k}} .
$$

Since $\langle\tau\rangle=-\tilde{f}^{\prime}(0),\left\langle\tau^{2}\right\rangle=\tilde{f}^{\prime \prime}(0)$ and $\left\langle\tau^{3}\right\rangle=-\tilde{f}^{(3)}(0)$, one concludes, differentiating equation (151), that $\langle\tau\rangle, r$ and $C$ are given, respectively, by equations (11), (3) and (5) with $\delta=\frac{\sum_{i=1}^{\mathcal{M}} \tau_{i}^{3}}{\left(\sum_{i=1}^{\mathcal{M}} \tau_{i}\right)^{3}}$. The form which $\tilde{f}_{\mathcal{M}}(s)$ takes for $\mathcal{M}=2,3$ even allows one to invert the Laplace transform $\tilde{l}(s)$ and obtain $l(u)$ in these two cases. One can therefore determine $\mathcal{C}(t)$ for arbitrary time $t$, using equation (47). This calculation is explicitly performed in Appendix A, as stated above.

In the above derivation, we have assumed that all moments of the distribution $f(\zeta)$ exist, in other words that the Laplace transform $\tilde{f}(s)$ is an analytic function at $s=0$. Such assumption is enterily justified when discussing the motion of a molecular motor in a homogeneous environment, as is the case of myosin $\mathrm{V}$ or kinesin. On the other hand, if one considers the motion of tracer particles in a rapidly rotating fluid [51], one is led to consider a renewal process with a waiting-time distribution with fat tails, which reflects the diverging sticking-times of the tracer particles. In a biological context, some of these results may also be applicable to the study of the motion of RNA-polymerase along a DNA-strand [7].

It is beyond our means to provide a complete discussion of this issue, However, one may consider a very simple model for a waiting-time distribution with fat tails, namely we take $f(\zeta)=\frac{(\nu-1) A^{\nu-1}}{(A+\zeta)^{\nu}}$, where $\nu>1$, such that $f(\zeta)$ is normalisable. For $2<\nu<3$, the assymptotic decay of $f(\zeta)$ is the same as that of a stable Lévy distribution [52. The Laplace transform of $f(\zeta)$ can be readily calculated, at least in the limit of small $s$, and one may carry through a significant number of calculations with this simple model.

One should start by considering the consequences of taking $\nu \leq 2$, in which case the distribution $f(\zeta)$ has an infinite first moment $\langle\tau\rangle$. In such a case, one can neither obtain equation (47) from equation (46) nor equation (6) from equation (59) (see below), since we are assuming that $\langle\tau\rangle$ is finite in the derivation of these results. Likewise, the equations that follow from equation (47), such as equation (48), and from equation (6), 
such as equation (7), are equally invalid. In physical terms, the assumption that fails is that one can consider the existence of a time-translation invariant regime, $t_{1}, t_{2} \gg\langle\tau\rangle$, for which the correlation functions $\left\langle\left(N\left(t_{1}\right)-N\left(t_{2}\right)\right)^{2}\right\rangle_{\text {conn }}$ and $S\left(q, t_{1}, t_{2}\right)$ depend only on $t=t_{1}-t_{2}$, since $\langle\tau\rangle$ is formally infinite.

In the case in which $\nu>2$, a time-translational regime does arise and our results hold through, except equation (49) which relies on the assumption that $\tilde{f}(s)$ is analytical at $s=0$. In particular, one may substitute the Laplace transform $\tilde{f}(s)$ in equation (47), and extract the asymptotic behaviour of $\mathcal{C}(t)$ at large $t$, by considering the limit of small $s$. The results we have obtained are simply a limiting case of those obtained by Weeks et al. [51], and we will therefore only quote the final result.

One can show that at large $t, \mathcal{C}(t)$ has the following asymptotic behaviour

$$
\mathcal{C}(t) \sim\left\{\begin{array}{c}
t^{4-\nu} \text { if } 2<\nu<3 \\
t \ln t \text { if } \nu=3 \\
\frac{r}{\langle\tau\rangle} t \text { if } \nu>3
\end{array},\right.
$$

with $r=\frac{\nu-1}{\nu-3}>1$ being the motor's (finite) randomness coefficient if $\nu>3$.

The result obtained in the parameter region $2<\nu<3$, in which the second moment of the distribution, or $r$, is formally infinite, can be interpreted as a superdiffusive behaviour of the tracer particle. Such a behaviour is also present in the model studied by Kafri and co-workers [7, 53].

We now consider the derivation of the Laplace transform of the density-density correlation function $S\left(q, t_{1}, t_{2}\right)=\left\langle e^{-i q d\left(N\left(t_{1}\right)-N\left(t_{2}\right)\right)}\right\rangle$ in the long time limit $t_{1}, t_{2} \rightarrow \infty$, which is given in equation (6). Such a correlation function can be obtained from the general expression for the probability-generating functional $\mathcal{Z}[j]$ by a judicious choice of $g(u)$ and of the source function $j(x)$ in equations (2224). If we take $g(u)=\theta(u)$ and

$$
j(x)=q d\left(\delta\left(x-t_{2}\right)-\delta\left(x-t_{1}\right)\right)
$$

then it is easy to see that $\mathcal{Z}[j]$ reduces to $S\left(q, t_{1}, t_{2}\right)$ for this particular choice of the functions $g(u)$ and $j(x)$. It also follows from equation (35) that $S\left(q, t_{1}, t_{2}\right)$ obeys the following integral equation

$$
S\left(q, t_{1}, t_{2}\right)=1+\left(e^{-i q d}-1\right) \int_{0}^{t} d \zeta l\left(q, t_{2}+\zeta\right)
$$

where $t=t_{1}-t_{2}$ and where $l\left(q, t_{2}+\zeta\right)$ is given by the solution of the integral equation

$$
l\left(q, t_{2}+\zeta\right)=l\left(t_{2}+\zeta\right)+\left(e^{-i q d}-1\right) \int_{0}^{\zeta} d \eta l(\zeta-\eta) l\left(q, t_{2}+\eta\right) \text { if } 0 \leq \zeta<t .
$$

This equation follows from (34) for the particular choice we made for $g(u)$ and $j(x)$. Such a system of equations can be solved using Laplace transformation. Following Godreche and Luck [27, we define the following Laplace transforms

$$
\begin{aligned}
& \tilde{l}\left(s ; t_{2}\right)=\int_{0}^{\infty} d \zeta e^{-s \zeta} l\left(t_{2}+\zeta\right), \\
& \tilde{l}\left(q, s ; t_{2}\right)=\int_{0}^{\infty} d \zeta e^{-s \zeta} l\left(q, t_{2}+\zeta\right), \\
& \tilde{S}\left(q, s ; t_{2}\right)=\int_{0}^{\infty} d t e^{-s t} S\left(q, t_{2}+t, t_{2}\right) .
\end{aligned}
$$


Substituting equations (5455) in (5758), one obtains, after some trivial manipulations, the following result for $\tilde{S}\left(q, s ; t_{2}\right)$

$$
\tilde{S}\left(q, s ; t_{2}\right)=\frac{1}{s}\left(1+\frac{e^{-i q d}-1}{1+\left(1-e^{-i q d}\right) \tilde{l}(s)} \tilde{l}\left(s ; t_{2}\right)\right)
$$

where $\tilde{l}(s)$ is the Laplace transform of $l(u)$, given by (20). In the long time limit $t_{2} \rightarrow \infty$, $l\left(t_{2}+\zeta\right) \rightarrow \frac{1}{\langle\tau\rangle}$ and $\tilde{l}\left(s ; t_{2}\right)=\frac{1}{\langle\tau\rangle s}$, which is independent of $t_{2}$. Therefore, $\tilde{S}\left(q, s ; t_{2}\right)$ is also independent of $t_{2}$ and we write it simply as $\tilde{S}(q, s)$ in this limit. Substituting $\tilde{l}(s)$ by its expression in terms of $\tilde{f}(s)$, as given by equation (20), in equation (59), we conclude that $\tilde{S}(q, s)$ is given by equation (6) [54].

We will now consider the relation between the Fourier and the Laplace transforms of $S(q, t)$. From its definition, one immediately concludes that for $t>0$,

$$
S(q,-t)=S(-q, t)=\overline{S(q, t)} .
$$

If we substitute this identity in the definition of $S(q, \omega)$, we obtain

$$
\begin{aligned}
& S(q, \omega)=\int_{-\infty}^{0} d t e^{i \omega t} S(q, t)+\int_{0}^{\infty} d t e^{i \omega t} S(q, t) \\
& =\overline{\int_{0}^{\infty} d t e^{i \omega t} S(q, t)}+\int_{0}^{\infty} d t e^{i \omega t} S(q, t) \\
& =2 \operatorname{Re} \tilde{S}(q, s=-i \omega),
\end{aligned}
$$

where we have performed the change of variable $t \rightarrow-t$ in the first term of the first line of (61). Equation (17) then follows from (6) and from (61). Finally, one obtains (8) by substituting $\tilde{f}(-i \omega)=(1-i \omega \tau)^{-1}$, valid for a simple Poisson process, in (7).

Before closing this section, we will briefly explain how one can obtain the expression for the spin-spin correlation function $\left\langle\vec{\sigma}_{t_{1}} \cdot \vec{\sigma}_{t_{2}}\right\rangle$ in the time domain in the cases in which the waiting-time distribution of the renewal process is given either by a single Poisson process, as in (11), or in which the waiting time-distribution is given by the convolution of two Poisson processes with the same characteristic time $\tau / 2$, as in (12), leaving the details to Appendix C. If the waiting-time distribution $f(\zeta)$ is a simple exponential, then, substituting the result for its Laplace transform $\tilde{f}(s)$ in equation (10), with $Q=3$, one observes that the Laplace transform of the spin-spin correlation function has a simple pole, and one can directly read $\left\langle\vec{\sigma}_{t_{1}} \cdot \vec{\sigma}_{t_{2}}\right\rangle$ from it. If the waiting-time distribution is given by the convolution of two Poisson processes with the same characteristic time, the Laplace transform of $\left\langle\vec{\sigma}_{t_{1}} \cdot \vec{\sigma}_{t_{2}}\right\rangle$, as given by equation (10), with $Q=3$, has two simple poles and this expression can always be written in terms of partial fractions involving one or the other of these poles. In that case, one can also read $\left\langle\vec{\sigma}_{t_{1}} \cdot \vec{\sigma}_{t_{2}}\right\rangle$ directly from it.

\section{Conclusion and outlook}

We have modelled the dynamics of a processive or rotary molecular motor as a renewal process, in line with the work of Svoboda and co-wokers. Using a functional technique, 
we have computed the mean-square deviation of the distance travelled by a processive motor and extracted its asymptotic limit at large times. For renewal processes composed of two or three Poisson substeps, we have computed this function outside the asymptotic regime, given the relevance of such processes for the study of myosin $\mathrm{V}$ and kinesin. It follows from our results that the measurement of this correlation function would permit one to extract additional information concerning the time constants which characterise the motor's chemical cycle.

We have also used the same functional method to compute the density-density correlation function of an ensemble of independent processive motors, which can be measured using the experimental techniques developed by Cappello and co-workers. We have also shown that in a particular limit such a function reduces to the spin-spin correlation function of a 'random-clock' model that has applications to the dynamics of rotary motors, such as F1-ATPase. The measurement of this correlation function, followed by its Fourier transformation to frequency space, would permit one, in both cases, to fit such a quantity to the theoretical results discussed above. Thus, one could obtain all the time constants characterising the molecular motor's chemical cycle, even in the case of chemical cycles composed of a large number of rate-limiting substeps.

As possible avenues of future research, one can indicate at least two experimental issues that still need to be addressed. The first experimental issue is the repetition of the experiments of Cappello and co-workers with an interference mask with a smaller period or the use of myosin $\mathrm{V}$, rather than kinesin, as the subject of study of such an experiment, which would allow the measurement of the density-density correlation function away from the limit of long wavelengths, where the motor simply behaves as a Brownian particle. The second issue is the direct measurement of the spin-spin correlation function in F1-ATPase using fluorescence microscopy and how one can improve the time resolution of the present technique.

In closing, we may say that the results presented in this paper can be used, in the different experimental contexts to which they apply, to provide for a precise fitting of the time constants associated with the rate-limiting steps of a molecular motor chemical cycle. It remains to be seen to what extent such information is of crucial importance to the understanding of the chemical kinetics of processive or rotary molecular motors, or if a qualitative understanding of the nature of the chemical cycle is by itself sufficient.

Acknowledgments: We acknowledge many helpful discussions with P. Pierobon, M. Badoual, A. Vilfan, G. Lattanzi, P. Benetatos, K. Kroy, R. Thul and G. Cappello. It is also a pleasure to acknowledge the referees of Physical Biology for their pertinent questions and comments, and the Style Editor of the journal, Valerie Parsegian, for her help with the subtleties of the English language in general and the improvement of the presentation in particular. J.E.S. and E.F. acknowledge financial support from the DFG in the framework of the Sonderforschungsbereich SFB 413/TP C6 and from IBM Deutschland in the framework of FZ Jülich TTB/1030.03.03 contract. A.P. acknowledges financial support from the Marie-Curie Fellowship no. HPMF-CT-2002- 
01529 and from the "Aides Jeunes Chercheurs" of the University of Montpellier 2, France. 


\section{Glossary}

Bead-motor assay: Experimental apparatus where a spherical plastic bead of micrometric size is coupled to a single molecular motor. The position of the bead can be controlled with high precision using two intense laser beams focused at a spot (known as optical tweezers). Due to its optical properties, the bead is attracted to the focus of the two beams and may be controlled at a distance.

Molecular motor stepping: Directed motion of a processive molecular motor (e.g. myosin V, kinesin, dynein) along a specific molecular track in the cell (e.g. actin in the case of myosin V, tubulin in the case of kinesin and dynein). Such a motion is composed of individual steps of fixed length, which occur at random times, determined by the chemistry of the process.

Probability-generating functional: Mathematical object which encodes in itself all the information that can be obtained (measured) from a random process. In the main text, we have computed the explicit form of such an object (39) for a renewal process where the interval distribution function was a simple exponential. From such an expression, one can obtain all the correlation function pertaining to such a renewal process. Thus, the ultimate goal of applied probability theory is the computation of the probability-generating functional for a given random process. All too often, one has to content oneself with well less than that.

Renewal process: Random counting process where an integer variable is increased by one unit at random times, the statistical distribution of the length of time intervals between sucessive counting events being a known function.

\section{Appendix A. Mean-square deviation of the displacement for a renewal process composed of two or three Poisson substeps}

In this appendix, we derive the explicit form, valid for arbitrary time $t$, of the meansquare deviation of the distance travelled by the motor $\mathcal{C}(t)$, for a renewal process whose waiting-time distribution is given by the convolution of two or three Poisson processes. As can been seen from equation (47), the knowledge of the renewal function $l(u)$ suffices to determine $\mathcal{C}(t)$.

We start with the simplest case, namely the case in which the waiting-time distribution is given by the convolution of two Poisson processes. In that case, the

Laplace transform of the waiting-time distribution is given by $\tilde{f}(s)=\frac{1}{\left(1+s \tau_{1}\right)\left(1+s \tau_{2}\right)}$, as follows from equation (51). Substituting this result in equation (20), one can show that the Laplace transform of the renewal function can be written in terms of partial fractions as

$$
\tilde{l}(s)=\frac{1}{\tau_{1}+\tau_{2}}\left(\frac{1}{s}-\frac{1}{s+1 / \tau_{1}+1 / \tau_{2}}\right) .
$$

From this equation, one can directly determine the inverse transform $l(u)$, which is given 
by

$$
l(u)=\frac{1-e^{-\left(\frac{1}{\tau_{1}}+\frac{1}{\tau_{2}}\right) u}}{\tau_{1}+\tau_{2}},
$$

which tends to $l(u) \rightarrow \frac{1}{\tau_{1}+\tau_{2}}$ in the limit of large $u$, in agreement with Blackwell's renewal theorem. Substituting this result for $l(u)$ in equation (47) and performing the integration over $u$, one obtains for $\mathcal{C}(t)$ the result

$$
\mathcal{C}(t)=\frac{r t}{\tau_{1}+\tau_{2}}+\frac{2 \tau_{1}^{2} \tau_{2}^{2}}{\left(\tau_{1}+\tau_{2}\right)^{4}}\left(1-e^{-\left(\frac{1}{\tau_{1}}+\frac{1}{\tau_{2}}\right) t}\right)
$$

where $r=\frac{\tau_{1}^{2}+\tau_{2}^{2}}{\left(\tau_{1}+\tau_{2}\right)^{2}}$ is the randomness parameter for a motor whose chemical cycle is composed of two rate-limiting steps. It can be easily checked that this function has the correct asymptotic forms at small and large $t$, has given by (44). We have plotted this function in figure 11, with $\tau_{1}=\tau_{2}=1 / 2$, i.e. when $r=1 / 2$.

In the case of a renewal processes whose waiting-time distribution is given by the convolution of three Poisson processes, it follows from (51) that $\tilde{f}(s)=$ $\frac{1}{\left(1+s \tau_{1}\right)\left(1+s \tau_{2}\right)\left(1+s \tau_{3}\right)}$. Substituting this result in equation (20), one can show that the Laplace transform of the renewal function can be written as

$$
\tilde{l}(s)=\frac{1}{\tau_{1}+\tau_{2}+\tau_{3}}\left[\frac{\gamma_{+}}{\gamma_{+}-\gamma_{-}}\left(\frac{1}{s}-\frac{1}{s+\gamma_{-}}\right)-\frac{\gamma_{-}}{\gamma_{+}-\gamma_{-}}\left(\frac{1}{s}-\frac{1}{s+\gamma_{+}}\right)\right],
$$

where $\gamma_{ \pm}=\frac{1}{2}\left[\left(\frac{1}{\tau_{1}}+\frac{1}{\tau_{2}}+\frac{1}{\tau_{3}}\right) \pm \sqrt{\left(\frac{1}{\tau_{1}}+\frac{1}{\tau_{2}}+\frac{1}{\tau_{3}}\right)^{2}-4\left(\frac{1}{\tau_{1} \tau_{2}}+\frac{1}{\tau_{1} \tau_{3}}+\frac{1}{\tau_{2} \tau_{3}}\right)}\right]$. One can also directly determine the inverse transform $l(u)$ from this equation, which is given by

$$
l(u)=\frac{1}{\tau_{1}+\tau_{2}+\tau_{3}}\left(\frac{\gamma_{+}}{\gamma_{+}-\gamma_{-}}\left(1-e^{-\gamma_{-} u}\right)-\frac{\gamma_{-}}{\gamma_{+}-\gamma_{-}}\left(1-e^{-\gamma_{+} u}\right)\right) .
$$

This function tends to $l(u) \rightarrow \frac{1}{\tau_{1}+\tau_{2}+\tau_{3}}$ in the limit of large $u$, which also agrees with Blackwell's renewal theorem. Substituting this result for $l(u)$ in equation (47) and performing the integration over $u$, one obtains for $\mathcal{C}(t)$ the result

$$
\begin{aligned}
\mathcal{C}(t)= & \frac{r t}{\tau_{1}+\tau_{2}+\tau_{3}}+\frac{2}{\left(\tau_{1}+\tau_{2}+\tau_{3}\right)^{2}}\left(\frac{\gamma_{+}}{\gamma_{-}^{2}\left(\gamma_{+}-\gamma_{-}\right)}\left(1-e^{-\gamma_{-} t}\right)\right. \\
& \left.-\frac{\gamma_{-}}{\gamma_{+}^{2}\left(\gamma_{+}-\gamma_{-}\right)}\left(1-e^{-\gamma_{+} t}\right)\right)
\end{aligned}
$$

where $r=\frac{\tau_{1}^{2}+\tau_{2}^{2}+\tau_{3}^{2}}{\left(\tau_{1}+\tau_{2}+\tau_{3}\right)^{2}}$ is the randomness parameter for a motor whose chemical cycle is composed of three rate-limiting steps. It can be easily checked that this function has the correct asymptotic forms at small and large $t$, has given by (44). We have plotted this function in figure 2, with $\tau_{1}=\tau_{2}=\tau_{3}=1 / 3$, i.e. when $r=1 / 3$.

\section{Appendix B. Density-density correlation function in Fourier space for a renewal process composed of two or three Poisson substeps}

All one has to do is to substitute the appropriate form for $\tilde{f}(-i \omega)$ in equation (17). For a renewal process whose waiting-time distribution is given by the convolution of two 
Poisson processes, $\tilde{f}(-i \omega)=\frac{1}{\left(1-i \omega \tau_{1}\right)\left(1-i \omega \tau_{2}\right)}$ and $S(q, \omega)$ is given by

$$
S(q, \omega)=\frac{2\left(\tau_{1}+\tau_{2}\right)(1-\cos (q d))\left(r+\alpha \omega^{2}\right)}{\left(\omega\left(\tau_{1}+\tau_{2}\right)-\sin (q d)\right)^{2}+\left(1-\cos (q d)-\tau_{1} \tau_{2} \omega^{2}\right)^{2}},
$$

where $r=\frac{\tau_{1}^{2}+\tau_{2}^{2}}{\left(\tau_{1}+\tau_{2}\right)^{2}}$ and $\alpha=\frac{\tau_{1}^{2} \tau_{2}^{2}}{\left(\tau_{1}+\tau_{2}\right)^{2}}$.

For a renewal process whose waiting-time distribution is given by the convolution of three Poisson processes, $\tilde{f}(-i \omega)=\frac{1}{\left(1-i \omega \tau_{1}\right)\left(1-i \omega \tau_{2}\right)\left(1-i \omega \tau_{3}\right)}$ and $S(q, \omega)$ is given by

$$
S(q, \omega)=2\left(\tau_{1}+\tau_{2}+\tau_{3}\right)(1-\cos (q d))\left(r+\alpha \omega^{2}+\beta \omega^{4}\right) / N
$$

where

$$
\begin{aligned}
N= & {\left[\omega\left(\tau_{1}+\tau_{2}+\tau_{3}\right)-\sin (q d)-\tau_{1} \tau_{2} \tau_{3} \omega^{3}\right]^{2} } \\
& +\left[1-\cos (q d)-\left(\tau_{1} \tau_{2}+\tau_{1} \tau_{3}+\tau_{2} \tau_{3}\right) \omega^{2}\right]^{2},
\end{aligned}
$$

and $r=\frac{\tau_{1}^{2}+\tau_{2}^{2}+\tau_{3}^{2}}{\left(\tau_{1}+\tau_{2}+\tau_{3}\right)^{2}}, \alpha=\frac{\tau_{1}^{2} \tau_{2}^{2}+\tau_{1}^{2} \tau_{3}^{2}+\tau_{2}^{2} \tau_{3}^{2}}{\left(\tau_{1}+\tau_{2}+\tau_{3}\right)^{2}}$ and $\beta=\frac{\tau_{1}^{2} \tau_{2}^{2} \tau_{3}^{2}}{\left(\tau_{1}+\tau_{2}+\tau_{3}\right)^{2}}$.

\section{Appendix C. Spin-spin correlation function for a renewal process composed of two Poisson substeps}

We first obtain the spin-spin correlation function in the time domain, as given by equation (12), if the two time constants are equal, i.e. if $\tau_{1}=\tau_{2}=\tau / 2$. In such a case, the Laplace transform of the waiting-time distribution is given by $\tilde{f}(s)=\frac{1}{(1+s \tau / 2)^{2}}$. Substituting such a result in equation (10), one can write the Laplace transform of the spin-spin correlation function in terms of partial fractions as

$$
\mathcal{L}\left\langle\vec{\sigma}_{t_{1}} \cdot \vec{\sigma}_{t_{2}}\right\rangle=\operatorname{Re}\left[\frac{3}{4} \frac{1}{s+\frac{2}{\tau}\left(1-e^{-i \pi / 3}\right)}+\frac{1}{4} \frac{1}{s+\frac{2}{\tau}\left(1+e^{-i \pi / 3}\right)}\right] .
$$

From such a result, one can immediatelly read its inverse Laplace transform, which is given by equation (12). Note that if the renewal process is a simple Poisson process, $\tilde{f}(s)=\frac{1}{1+s \tau}$ and the Laplace transform of the spin-spin correlation function involves a simple pole, which make its inversion trivial and we obtain (11).

For a general choice of the time constants $\tau_{1}$ and $\tau_{2}$, one can also write the Laplace transform of the spin-spin correlation function in terms of partial fractions, which permits to invert such a transform. However, extracting the real part of such an expression becomes a cumbersome exercise, albeit a trivial one. It is simpler, also from the viewpoint of the experimental fitting of the data, to consider instead the Fourier transform of the spin-spin correlation function, which is defined as $F T\left\langle\vec{\sigma}_{t_{1}} \cdot \vec{\sigma}_{t_{2}}\right\rangle=$ $\int_{-\infty}^{+\infty} d t e^{i \omega t}\left\langle\vec{\sigma}_{t_{1}} \cdot \vec{\sigma}_{t_{2}}\right\rangle$, with $t=t_{1}-t_{2}$. Since one can write the spin-spin correlation function as $\left\langle\vec{\sigma}_{t_{1}} \cdot \vec{\sigma}_{t_{2}}\right\rangle=\frac{1}{2}(S(2 \pi / 3, t)+S(2 \pi / 3,-t))$ (with $d=1$ ), the Fourier transform of this function is given by

$$
F T\left\langle\vec{\sigma}_{t_{1}} \cdot \vec{\sigma}_{t_{2}}\right\rangle=\frac{1}{2}(S(2 \pi / 3, \omega)+S(2 \pi / 3,-\omega)) .
$$


where $S(q=2 \pi / 3, \omega)$ is given by (B.1), valid for a renewal process composed of two Poisson substeps. We finally obtain

$$
\begin{aligned}
F T\left\langle\vec{\sigma}_{t_{1}} \cdot \vec{\sigma}_{t_{2}}\right\rangle= & \frac{3}{2}\left(\tau_{1}+\tau_{2}\right)\left(r+\alpha \omega^{2}\right)\left[\frac{1}{\left(\omega\left(\tau_{1}+\tau_{2}\right)-\sqrt{3} / 2\right)^{2}+\left(3 / 2-\tau_{1} \tau_{2} \omega^{2}\right)^{2}}\right. \\
& \left.+\frac{1}{\left(\omega\left(\tau_{1}+\tau_{2}\right)+\sqrt{3} / 2\right)^{2}+\left(3 / 2-\tau_{1} \tau_{2} \omega^{2}\right)^{2}}\right], \quad \text { (C.3) }
\end{aligned}
$$

where $r=\frac{\tau_{1}^{2}+\tau_{2}^{2}}{\left(\tau_{1}+\tau_{2}\right)^{2}}$ and $\alpha=\frac{\tau_{1}^{2} \tau_{2}^{2}}{\left(\tau_{1}+\tau_{2}\right)^{2}}$. We plotted this function for three different choices of the time constants $\tau_{1}$ and $\tau_{2}$, such that $\tau_{1}+\tau_{2}=1$, in figure 4 . 


\section{References}

[1] M. Schliwa (ed.). Molecular Motors. Wiley VCH, 2002.

[2] The best analogy one can make between a molecular motor and a macroscopic thermodynamic engine would be to a fuel-cell coupled to an electric engine, operating at constant temperature. However, such an analogy is far from being perfect, since in such an arrangement the generation of an electric current and its convertion into motion occur in physically distinct regions, whereas in a molecular motor the conformational changes of the molecule, which engender its overall motion, occur directly due to chemical transformations.

[3] F. Juelicher. Force and motion generation of molecular motors: A generic description. In Transport and Structure: Their Competitive Roles in Biophysics and Chemistry, Lecture Notes in Physics. Springer, 1999.

[4] M. Rief, R. S. Rock, A. D. Mehta, M. S. Mooseker, R. E. Cheney, and J. A. Spudich. Myosin-v stepping kinetics: A molecular model for processivity. Proc. Natl. Acad. Sci. (USA), 97:9482$9486,2000$.

[5] W. Schief and J. Howard. Conformational changes during kinesin motility. Current Opinion in Cell Biology, 13:19-28, 2001.

[6] B. Alberts, D. Bray, J. Lewis, M. Raff, K. Roberts, and J. Watson. Molecular Biology of the Cell. Garland Publishing, 1994.

[7] Y. Kafri, D. Lubensky, and D. Nelson. Dynamics of molecular motors and polymer translocation with sequence heterogeneity. Biophys. J., 86:3373-3391, 2004.

[8] M. Schnitzer and S. Block. Kinesin hydrolyses one atp per 8-nm step. Nature, 388:386-390, 1997.

[9] A. Mehta, R. Rock, M. Rief, J. Spudich, M. Mooseker, and R. Cheney. Myosin-v is a processive actin-based motor. Nature, 400:590-593, 1999.

[10] M. Ali, S. Uemura, K. Adachi, H. Itoh, K. Kinosita, and S. Ishiwata. Myosin v is a left-handed spiral motor on the right-handed actin helix. Nature Structural Biology, 9:464-467, 2002.

[11] In reality, the step-size of $34.8 \mathrm{~nm}$ is just an average value, since myosin $\mathrm{V}$ may perform steps involving the attachment of the free head to the 11th or 13th unit (with respect to that of the bound head) along the actin spiral[10. The description of the motion of myosin $\mathrm{V}$ in terms of a renewal process with a constant step size is thus just an approximation, but which should be valid for length scales (times) much larger than the distance of $5.5 \mathrm{~nm}$ (crossing-time) between the neighbouring units.

[12] M. Yoshida, E. Muneyuki, and T. Hisabori. Atp synthase-a marvellous rotary engine of the cell. Nature Reviews, 2:669-677, 2001.

[13] P. Boyer. The atp synthase: a splendid molecular machine. Annu. Rev. Biochem., 67:717-749, 1997.

[14] K. Svoboda, P. Mitra, and S. Block. Fluctuation anylysis of motor protein movement and single enzyme kinetics. Proc. Natl. Acad. Sci. (USA), 91:11782-11786, 1994.

[15] A. Mehta, M. Rief, J. Spudich, D. Smith, and R. Simmons. Single-molecule biomechanics with optical methods. Science, 283:1689-1695, 1999.

[16] A. Mehta. Myosin learns to walk. J. Cell Science, 114:1981-1998, 2001.

[17] C. Veigel, F. Wang, M. Bartoo, J. Sellers, and J. Molloy. The gated gait of the processive molecular motor, myosin v. Nature Cell Biology, 4:59-65, 2002.

[18] S. Block, C. Ashbury, J. Shaevitz, and M. Lang. Probing the kinesin reaction cycle with a 2d optical force clamp. Proc. Natl. Acad. Sci. (USA), 100:2351-2356, 2003.

[19] M. Schnitzer, K. Visscher, and S. Block. Force production by single kinesin motors. Nature Cell Biology, 2:718-723, 2000.

[20] G. Cappello, M. Badoual, A. Ott, J. Prost, and L. Busoni. Kinesin motion in the absence of external forces characterized by interference total internal reflection microscopy. Phys. Rev. E, 68:021907-7, 2003.

[21] M. Badoual, G. Cappello, K. Zeldovich, and J. Prost. Simulations of interference total internal 
reflection microscopy experiments with processive molecular motors. unpublished.

[22] K. Adachi, R. Yasuda, H. Noji, H. Itoh, Y. Harada, M. Yoshida, and K. Kinosita. Stepping rotation of f1-atpase visualized through angle-resolved single-fluorophore imaging. Proc. Natl. Acad. Sci. (USA), 97:7243-7247, 2000.

[23] K. Kinosita, K. Adachi, and H. Itoh. Rotation of f1-atpase: How an atp-driven molecular machine may work. Annu. Rev. Biophys. Biomol. Struct., 33:245-268, 2004.

[24] R. Glauber. Quantum theory of coherence. In Quantum Optics, pages 53-125. Academic Press, 1970.

[25] C. Benard and O. Macchi. Detection and 'emission' processes of quantum particles in a 'chaotic state'. J. Math. Phys., 14:155-167, 1973.

[26] V. Apanasovich and S. Paltsev. Distortion of photon-correlation functions in detection systems with paralyzable dead-time effects. J. Opt. Soc. Am., 9:1550-1554, 1995.

[27] C. Godreche and J. Luck. Statistics of the occupation time of renewal processes. J. Stat. Phys., 104:489-524, 2001.

[28] P. Allegrini, G. Aquino, P. Grigolini, L. Palatella, A. Rosa, and B. West. Correlation function and generalized master equation of arbitrary age. condmat-0409600, 2004.

[29] I. Giardina, J.P. Bouchaud, and M. Mézard. Microscopic models for long ranged volatility correlations. In Physica A. Proceedings of the NATO Advanced Research Workshop 'Application of Physics to Economic Modelling', volume 299, pages 28-39. 2001.

[30] D. Daley and D. Vere-Jones. An Introduction to the Theory of Point Processes, volume I: Elementary Theory and Methods. Springer, 2003.

[31] J. Honerkamp. Stochastic Dynamical Systems - Concepts, Numerical Methods, Data Analysis: Theory, Instrumentation, and Applications. John Wiley and Sons, 1994.

[32] D. Cox. Renewal Theory. Methuen and Co, 1962.

[33] A. Hoenger, M. Thormaehlen, R. Diaz-Avalos, M. Doerhoefer, K. Goldie, J. Mueller, and E. Mandelkow. A new look at the microtubule binding patterns of dimeric kinesins. J. Mol. Biol., 297:1087-1103, 2000.

[34] F. Juelicher, A. Ajdari, and J. Prost. Modeling molecular motors. Rev. Mod. Phys., 69:1269-1281, 1997.

[35] A. Parmeggiani, F. Juelicher, A. Ajdari, and J. Prost. Energy transduction of isothermal ratchets: Generic aspects and specific examples close to and far from equilibrium. Phys. Rev. E, 60:21272140, 1999.

[36] A. Parmeggiani, F. Juelicher, L. Peliti, and J. Prost. Detachment of molecular motors under tangential loading. Europhys. Lett., 56:603-609, 2001.

[37] G. Lattanzi and A. Maritan. Master equation approach to molecular motors. Phys. Rev. E, 64:061905, 2001.

[38] M. Fisher and A. Kolomeisky. Simple mechanochemistry describes the dynamics of kinesin molecules. Proc. Natl. Acad. Sci. (USA), 98:7748-7753, 2001.

[39] A. Kolomeisky and M. Fisher. A simple kinetic model describes the processivity of myosin-v. Biophys. J., 84:1642-1650, 2003.

[40] R. Lipowsky and N. Jaster. Molecular motor cycles: From ratchets to networks. J. Stat. Phys., 110:1141-1167, 2003.

[41] A.Vilfan. Elastic lever arm for myosin v. Biophys. J., 88:3792-3805, 2005.

[42] The randomness coefficient is nothing more than the inverse of the Peclet number $\propto d v / D$, used in the study of polydisperse systems, where $d$ are the typical dimensions of the object, $v$ its average velocity and $D$ its diffusion constant.

[43] When we say that a renewal process is composed of $\mathcal{M}$ rate-limiting processes, what we of course mean is that the waiting-time distribution $f(\zeta)$ of such a process is given by the convolution of $\mathcal{M}$ simple Poisson processes, with characteristic times $\tau_{1}, \cdots, \tau_{\mathcal{M}}$, in line with the model introduced by Svoboda and coworkers. We believe that the number of words spared in the text justifies commiting such a small imprecision. 
[44] The randomness parameter can be larger than one, e. g. if one allows for the presence of thermal noise, through the Brownian motion of the dielectric bead, or if the motor can perform backward steps. The first effect can be accounted for if one assumes that such noise is uncorrelated with the renewal process ocurring in the motor [14, 21] and contributes additively to the motion of the bead. The second effect is only pronounced if one is probing the motor close to the stall force or working at very low ATP concentration.

[45] The turnover time can also be determined by the measurement of $\mathcal{C}(t)$ at small times $t$, but such a measurement is likely to be much less precise than the measurement of $\langle\tau\rangle$ obtained from the average displacement of the motor, which extends itself through several decades.

[46] Strictly speaking, the quantity measured in the experiments of Cappello and co-workers 20, 21] is the intensity-intensity correlation function of the light scattered by the bead, which is given by the real part of $S(q, t)$, with $q=2 \pi / \Lambda$, where $\Lambda$ is the length of the mask used to create the interference pattern. Therefore, one has that the Fourier transform of $\operatorname{Re} S(q, t)$ is in fact given by $\frac{1}{2}(S(q, \omega)+S(q,-\omega))$, as in the case of the spin-spin correlation function, see below in the text.

[47] Since $S(q, \omega)$ reduces to equation (9) at small $q$ and $\omega$, it is advisable that experiments such as those carried by Cappello and co-workers [20, 21, in which the wave-vector $q=2 \pi / \Lambda$ is kept constant throughout the experiment, are performed at high values of $q$, or small values of the mask length $\Lambda$. Otherwise, if $q d \ll 1$, one will not be able to determine more than the two parameters $v$ and $D$, which characterise the motor's Brownian motion in the large wavelength limit. In the experiments reported in reference [20], the mask length $\Lambda=190 \mathrm{~nm}$, whereas $d$, the step size of kinesin, the motor whose motion was studied, is equal to $8 \mathrm{~nm}$. One thus has that $q d \approx 0.265$, i.e. one is still in the limit of low $q$ and one will not be able to fully determine the characteristic times pertaining to the three or four rate-limiting steps of the chemical cycle of kinesin. Since for myosin $\mathrm{V}, d=35 \mathrm{~nm}$, such experiments will probably yield clearer results if performed with this molecular motor.

[48] For the three different levels of polarisation measured by Adachi and co-workers [22, the signs in the expressions for $(h(T), v(T))$, should be, for levels a, b, c, respectively $(1,1),(-1,1),(-1,-1)$.

[49] We would explicitly like to thank the second referee of Physical Biology for having pointed out the possibility to carry out the measurement of the spin-spin correlation function using this method.

[50] O. Macchi. Stochastic processes and multicoincidences. IEEE Transactions on Information Theory, 17:2-7, 1971.

[51] E. Weeks, J. Urbach, and H. Swinney. Anomalous diffusion in asymmetric random walks with a quasi-geostrophic flow example. Physica D, 97:291-310, 1996.

[52] J. P. Bouchaud and M. Potters. Théorie des Risques Financiers. Commissariat à l'Énergie Atomique, 1997.

[53] We would explicitly like to thank the first referee of Physical Biology for having raised the issue of a waiting-time distribution with fat tails.

[54] One can obtain equation (6) directly, without having to consider the long time limit $t_{2} \rightarrow \infty$, if one chooses the waiting-time distribution for the first step to be given not by $f(\zeta)$, but by $\mathcal{F}(\zeta)=\frac{1}{\langle\tau\rangle} \int_{\zeta}^{\infty} d \eta f(\eta)[\underline{50}$. Such an initial distribution completely kills off all the transients and garanties that the time-translation invariant regime is valid right after $t_{2}=0$. In fact, the choice of such a distribution can reduce the computation of multiple-time correlations functions such as $\mathcal{C}(t)$ to the computation of single-time correlation functions. However, in a real experiment performed with a molecular motor, there is no guaranty that the waiting-time distribution for the first step is actually given by $\mathcal{F}(\zeta)$ and therefore it is crucial that the measurements, if they are not to be influenced by initial transients which may even vary from one experiment to the next, are taken in the limit $t_{1}, t_{2} \gg\langle\tau\rangle$, i.e. after the motor has completed several steps (chemical cycles). We have therefored chosen not to consider this particular case in our discussion. 